\title{
Analytic High-Order Energy Derivatives for Metal Nanoparticle-Mediated Infrared and Raman Scattering Spectra within the Framework of Quantum Mechanics/Molecular Mechanics Model with Induced Charges and Dipoles
}

\author{
Zheng Pei, ${ }^{1}$ Yuezhi Mao, ${ }^{2, \text { a) }}$ Yihan Shao, ${ }^{3}$, b) and WanZhen Liang ${ }^{1, c)}$ \\ ${ }^{1)}$ State Key Laboratory of Physical Chemistry of Solid Surfaces, Collaborative Innovation Center of Chemistry for Energy Materials, \\ Fujian Provincial Key Laboratory of Theoretical and Computational Chemistry, and Department of Chemistry, \\ College of Chemistry and Chemical Engineering, Xiamen University, Xiamen 361005, \\ P. R. China. \\ 2) Department of Chemistry, Stanford University, Stanford, CA 94305, United States. \\ ${ }^{3)}$ Department of Chemistry and Biochemistry, University of Oklahoma, Norman, OK 73019 , \\ United States.
}

This work is devoted to deriving and implementing analytic second- and third-order energy derivatives with respect to the nuclear coordinates and external electric field within the framework of the hybrid quantum mechanics/molecular mechanics method with induced charges and dipoles (QM/DIM). Using these analytic energy derivatives, one can efficiently compute the harmonic vibrational frequencies, infrared (IR) and Raman scattering (RS) spectra of the molecule in the proximity of noble metal clusters/nanoparticles. The validity and accuracy of these analytic implementations are demonstrated by the comparison of results obtained by the finite-difference method and the analytic approaches, and by the full QM and QM/DIM calculations. The complexes formed by pyridine and two sizes of gold clusters $\left(\mathrm{Au}_{18}\right.$ and $\left.\mathrm{Au}_{32}\right)$ at varying intersystem distances of 3, 4, and $5 \AA$ are used as the test systems, and Raman spectra of 4,4'-bipyridine in the proximity of $\mathrm{Au}_{2057}$ and $\mathrm{Ag}_{2057}$ metal nanoparticles (MNP) are calculated by QM/DIM method and compared with experimental results as well. We find that the QM/DIM model can well reproduce the IR spectra obtained from full QM calculations for all the configurations, while though it properly enhances some of the vibrational modes, it artificially overestimates RS spectral intensities of several modes for the systems with very short intersystem distance. We show that this could be improved, however, by incorporating the hyperpolarizability of the gold metal cluster in the evaluation of RS intensities. Additionally, we address the potential impact of charge migration between the adsorbate and MNPs.

\section{INTRODUCTION}

In the last several decades, plasmonic metal nanoparticles (MNPs) have generated a great amount of interest due to the dramatic effect of surface localized plasmon resonance. ${ }^{1-4}$ Currently, plasmonic MNPs have been widely used to control and manipulate light at the nanoscale, thus regulating the photophysical and photochemical properties of molecules in their vicinity, such as enhancing molecular optical signals (absorption, ${ }^{5}$ Raman, ${ }^{6,7}$ and fluorescence ${ }^{8-10}$ ) and mediating molecular photochemical reactions. ${ }^{1-14}$ Meanwhile, many theoretical and computational methods were developed to unravel the detailed mechanisms of molecular spectral enhancements, ${ }^{15}$ plasmon-mediated chemical reactions, ${ }^{16,17}$ as well as plasmon-enhanced resonance energy transfer. ${ }^{18-21}$ In many cases, the plasmon enhancement arises mainly from the electromagnetic mechanism, where an external electric field can be substantially magnified by an MNP around its surface. Accordingly, several classical nonatomistic methods ${ }^{22}$ had been widely applied.

The hybrid MNPs-molecular systems represent a challenge for theoretical and computational methods because the con-

\footnotetext{
a)Electronic mail: yuezhi.mao@stanford.edu

b) Electronic mail: yihan.shao@ou.edu

c)Electronic mail: liangwz@xmu.edu.cn
}

stituents can not be treated on the same footing by the stateof-the-art methods. The properties of molecules require a quantum mechanics (QM) description thanks to the high accuracy of QM methods. ${ }^{23,24}$ However, QM methods are unable to describe the medium to large-sized MNPs due to their steep computational cost. Two kinds of simplified treatments have been usually adopted. One is to simplify the problem by assuming the molecules bonded to very small metal clusters. ${ }^{25-32}$ With this treatment, the optical properties of molecule-metal cluster systems can be described by the QM methods. Small MNPs, however, don't support the bulk plasmon. The other is to adopt the mixed quantum/classical approach, which combines the QM approaches with classical mechanics or electrodynamics, such as the Mie theory, ${ }^{33}$ discrete dipole approximation, ${ }^{34}$ finite difference time domain, ${ }^{5}$ and polarizable continuum models. ${ }^{35-37}$

To more accurately capture the plasmonic effect from MNPs with different sizes, shapes, and compositions, however, atomistic modeling of the MNP-adsorbate system is needed. Specifically, to account for the large polarizability of MNPs, (induced) charges, dipoles, and/or even multipoles would be introduced at each atom site, leading to several (polarizable) force field models for MNPs. These molecular mechanics (MM) methods include the point-dipole interaction model $^{38,39}$ (including its combination with either electronegativity equalization ${ }^{40}$ or charge-transfer ${ }^{41}$ ), charge-dipole interaction model, ${ }^{42,43}$ discrete interaction model (DIM) ${ }^{44,45}$ and its coordination-dependent variant (cd-DIM), ${ }^{46}$ and atomic 
dipole approximation model. ${ }^{47}$

These MM models can be employed in combined quantum mechanics/molecular mechanics (QM/MM) modeling of probe-MNP complexes, where the probe molecule constitutes the QM region so that its vibrational motions (in infrared and Raman spectroscopy) or electronic transitions (in fluorescence) are treated using ab initio QM theories. Mikkelsen et $a l$ have investigated the hyperpolarizability changes of organic molecules near gold nanoparticles by a polarizable QM/MM approach recently. ${ }^{48,49}$ The DIM model with induced charges and induced dipoles at each metal atom, in particular, has been combined with QM methods by Jensen and others ${ }^{50-54}$ to model MNP-mediated one-photon ${ }^{51,55}$ and two-photon absorption, ${ }^{56}$ Raman, ${ }^{52,57-59}$ and fluorescence spectra. ${ }^{60}$ In parallel to the QM/MM study of MNP-enhanced electronic transitions, Giovannini, Cappelli, and others have combined density functional theory (DFT) and time-dependent density functional theory (TDDFT) with their $\mathrm{FQF} \mu$ models for water molecules, which are also based on fluctuating charges and induced dipoles. ${ }^{61,62}$

Analytic energy derivatives can provide computational advantages in molecular geometry optimizations, vibrational frequency calculations, and molecular property descriptions. The analytic Raman intensities of the QM method have been derived and implemented decades ago. ${ }^{63-65}$ Recently, the implementations of analytic Hessian and high-order molecular properties (such as IR and Raman intensities) have been extended to several hybrid (polarizable) QM/MM schemes for solvents or protein systems. ${ }^{66-68}$ Here, inspired by the encouraging results of the QM/DIM model from Jensen et al, ${ }^{50-52}$ we present our implementation of this model and its analytic energy derivatives with respect to the nuclear coordinates and perturbed electric field for the study of the adsorbate-MNP systems. Compared to earlier implementations of IR and Raman intensities within a polarizable QM/MM model, ${ }^{66-68}$ our implementation is applicable to polarizable force fields involving both induced charges and dipoles, such as the DIM model for metal clusters.

The paper is organized as follows. Section II briefly recaps the basic theoretical foundation of the polarizable QM/MM model for the ground-state energy of a molecule-MNP system and presents the analytical expressions for high-order derivatives of the energy with respect to the QM nuclear and field perturbations. It is followed by the computational details in Section III. In Section IV, using the pyridine molecule in the vicinity of three gold clusters as testing systems, the computed IR and Raman spectra are presented and discussed. In addition, the SERS of 4,4'-bipyridine on gold and silver MNPs are simulated by QM/DIM method and compared with experimental spectra. Moreover, the effects of nonlinear response of MNP and charge migration to Raman intensity are analysed. Finally, the concluding remarks and potential future improvements are summarized in the last section.

\section{THEORY}

\section{II.A. Total Energy of Hybrid QM/MM System}

Here the DIM method is briefly described with a compact notation and the reader is referred to the original publications. ${ }^{44,45}$ The energy of the MM region is given by the electrostatic interaction,

$$
E^{\mathrm{MM}}=\frac{1}{2} \mathscr{M}^{\text {ind }} \cdot \mathscr{T} \cdot \mathscr{M}^{\text {ind }}-\mathscr{M}^{\text {ind }} \cdot \mathscr{F}^{\mathrm{tot}}
$$

where $\mathscr{M}^{\text {ind }}$ represents the collection of induced charges and dipoles $\left(q^{\text {ind }}, \vec{\mu}^{\text {ind }}\right)$ on MM atoms; $\mathscr{T}$ the interaction matrices of charge-charge, charge-dipole, and dipole-dipole $\left(T^{(0)}, T^{(1)}, T^{(2)}\right)$ as

$$
T_{i j}^{(0)}=\frac{1}{r_{i j}} \operatorname{erf}\left(\frac{r_{i j}}{\sigma}\right), \quad T_{i j}^{(n)}=\left(-\nabla_{j}\right)^{n} T_{i j}^{(0)}
$$

where $r_{i j}$ is the distance between the $i$-th and $j$-th MM atoms, and $\sigma$ is the width of Gaussian functions used to damp the electrostatic interaction between neighboring atoms. ${ }^{44}$ The diagonal block of $\mathscr{T}$ is formed by the atomic capacitance $\left(c_{i}\right)$ and polarizability $\left(\alpha_{i}\right)$ for charge and dipole self-interactions at $i$-th MM atom, respectively. $\mathscr{F}^{\text {tot }}$ is the total external generalized field exerted on the MM sites, including both potentials and fields $(-V, \vec{E})$. Using the variational condition, the stationary solution is given by solving a linear equation,

$$
\left(\begin{array}{cc}
\mathscr{T} & \mathbf{1}^{\prime T} \\
\mathbf{1}^{\prime} & 0
\end{array}\right)\left(\begin{array}{c}
\mathscr{M}^{\text {ind }} \\
\lambda
\end{array}\right)=\left(\begin{array}{c}
\mathscr{F}^{\text {tot }} \\
Q
\end{array}\right)
$$

where the Lagrangian multiplier $\lambda$ is introduced to constrain the net charge for the MM region as $Q . \mathbf{1}^{\prime}$ refers to a row vector $(1,0,0,0,1,0,0,0, \cdots)$ with a value of 1 for only the induced charge on each $\mathrm{MM}$ atom. When $Q$ is equal to 0 (as for all test cases in this work), the solution to Eq. (3) could be formally written as,

$$
\mathscr{M}^{\text {ind }}=\mathscr{T}^{-1} \cdot \mathscr{F}^{\mathrm{tot}}
$$

where $\mathscr{T}^{-1}$ represents the upper-left $4 N_{\text {MM }} \times 4 N_{\text {MM }}$ block of the inverse of the matrix in Eq. (3). Therefore, the MM energy is reformulated as

$$
\begin{aligned}
E^{\mathrm{MM}} & =-\frac{1}{2} \mathscr{M}^{\mathrm{ind}} \cdot \mathscr{F}^{\mathrm{tot}}=-\frac{1}{2} \mathscr{M}^{\mathrm{ind}} \cdot \mathscr{T} \cdot \mathscr{M}^{\text {ind }} \\
& =-\frac{1}{2} \mathscr{F}^{\mathrm{tot}} \cdot \mathscr{T}^{-1} \cdot \mathscr{F}^{\mathrm{tot}}
\end{aligned}
$$

The last expression for the MM energy will be used in the derivation of the high-order derivatives of the energy.

When the MM region is adjacent to the QM region (as described on the DFT level of theory), the total generalized field $\mathscr{F}^{\text {tot }}$ would include the contributions from the QM region in addition to the external source,

$$
\begin{aligned}
\mathscr{F}^{\mathrm{tot}} & =\mathscr{F}^{\mathrm{QM}}+\mathscr{F}^{\mathrm{ext}} \\
& =-\mathbf{V} \cdot \mathbf{P}+\sum_{I} Z_{I} \mathscr{T}_{I}+\mathscr{R} \vec{f}^{\mathrm{ext}}
\end{aligned}
$$


where the matrix $\mathbf{V}$ contains integrals $\left\langle\phi_{\mu}|\mathscr{T}| \phi_{v}\right\rangle$. Namely, for each pair of basis function $\left(\phi_{\mu}\right.$ and $\left.\phi_{v}\right)$ and the $j$-th MM atom, it contains the interaction between the basis function pair and a Gaussian MM charge (with width $\sigma^{\prime}$ ),

$$
V_{j, \mu v}^{(0)}=\left\langle\phi_{\mu}\left|\frac{1}{\left|\vec{r}-\vec{R}_{j}^{\prime}\right|} \operatorname{erf}\left(\frac{\left|\vec{r}-\vec{R}_{j}^{\prime}\right|}{\sigma^{\prime}}\right)\right| \phi_{v}\right\rangle
$$

and its first derivatives.

$$
V_{j, \mu \nu}^{(1)}=-\nabla_{j}\left(\left\langle\phi_{\mu}\left|\frac{1}{\left|\vec{r}-\vec{R}_{j}^{\prime}\right|} \operatorname{erf}\left(\frac{\left|\vec{r}-\vec{R}_{j}^{\prime}\right|}{\sigma^{\prime}}\right)\right| \phi_{\nu}\right\rangle\right)
$$

In Eq. (6), $\mathbf{P}$ is the density matrix with elements $P^{\mu v}, Z_{I}$ labels the $I$-th QM nuclear charge and $\mathscr{T}_{I}$ is the corresponding generalized external field on MM atoms, and $\mathscr{R} \vec{f}^{\text {ext }}$ represents the generalized field on each MM atom at position $\left(R_{j x}^{\prime}, R_{j y}^{\prime}, R_{j z}^{\prime}\right)$ :

$$
\mathscr{R} \vec{f}^{\mathrm{ext}}=\left(\begin{array}{ccc}
R_{1 x}^{\prime} & R_{1 y}^{\prime} & R_{1 z}^{\prime} \\
1 & 0 & 0 \\
0 & 1 & 0 \\
0 & 0 & 1 \\
R_{2 x}^{\prime} & R_{2 y}^{\prime} & R_{2 z}^{\prime} \\
1 & 0 & 0 \\
0 & 1 & 0 \\
0 & 0 & 1 \\
\cdots & \cdots & \ldots
\end{array}\right)\left(\begin{array}{l}
f_{x}^{\mathrm{ext}} \\
f_{y}^{\text {ext }} \\
f_{z}^{\text {ext }}
\end{array}\right)
$$

With the definition of the total generalized field $\mathscr{F}^{\text {tot }}$ in Eq. (6), the total electronic energy of the system can then be expressed as

$$
\begin{aligned}
E= & \mathbf{P} \cdot\left(\mathbf{h}_{0}+\frac{1}{2} \mathbf{I I} \cdot \mathbf{P}\right)+E_{\mathrm{xc}}-\vec{\mu}^{\mathrm{QM}} \cdot \vec{f}^{\mathrm{ext}} \\
& -\frac{1}{2} \mathscr{M}^{\mathrm{ind}} \cdot \mathscr{F}^{\mathrm{tot}}+E_{\mathrm{vdW}}^{\mathrm{QM} / \mathrm{MM}}
\end{aligned}
$$

where the first term is the well-known DFT core Hamiltonian $\mathbf{h}_{0}$ and the two-electron integral supermatrix II in atomic basis $\left(\phi_{\mu}, \phi_{v}, \phi_{\lambda}, \phi_{\sigma}\right)$ is

$$
\Pi_{\mu v, \lambda \sigma}=(\mu v \mid \lambda \sigma)-C_{\mathrm{HFX}}\left(\mu \lambda\left|f\left(\vec{r}_{12}\right)\right| v \sigma\right)
$$

adopting approximate coefficient $C_{\mathrm{HFX}}$ and operator $f\left(\vec{r}_{12}\right)$ for hybrid and range-separated functionals. Further, $E_{\mathrm{xc}}=$ $\int \mathrm{d} \vec{r} f_{\mathrm{xc}}(\vec{r})$ is the DFT exchange-correction energy, and $\vec{\mu}^{\mathrm{QM}}$ is the dipole moment of the QM region,

$$
\vec{\mu}^{\mathrm{QM}}=-\mathbf{P} \cdot \overrightarrow{\mathbf{M}}+\sum_{I} Z_{I} \vec{R}_{I}
$$

which leads to the interaction between QM and external fields within the dipole approximation. Here $\overrightarrow{\mathbf{M}}$ is the dipole moment matrix and $\vec{R}_{I}$ the $I$-th QM nuclear coordinate. The corresponding Fock matrix to be used in the SCF cycles could be obtained by differentiating the total energy given by Eq. (10) with respect to the density matrix $(\mathbf{P})$ :

$$
\mathbf{F}=\mathbf{h}_{0}+\overrightarrow{\mathbf{M}} \cdot \vec{f}^{\mathrm{ext}}+\mathscr{M}^{\mathrm{ind}} \cdot \mathbf{V}+\mathbf{I I} \cdot \mathbf{P}+\mathbf{F}_{\mathrm{xc}}
$$

where the elements of $\mathbf{F}_{x c}$ can be computed as

$$
\left(F_{\mathrm{xc}}\right)_{\mu \nu}=\int \mathrm{d} \vec{r} \sum_{\xi} \frac{\partial f_{\mathrm{xc}}}{\partial \xi} \frac{\partial \xi}{\partial P^{\mu \nu}}
$$

with variables, $\xi$, being electron density or its gradient. ${ }^{69}$ With this Fock matrix, we could define a total core Hamiltonian as,

$$
\mathbf{h}=\mathbf{h}_{0}+\overrightarrow{\mathbf{M}} \cdot \vec{f}^{\mathrm{ext}}+\mathscr{M}^{\text {ind }} \mathbf{V}
$$

and then the expression

$$
\begin{aligned}
E= & \mathbf{P} \cdot\left(\mathbf{h}+\frac{1}{2} \mathbf{I I} \cdot \mathbf{P}\right)+E_{\mathrm{xc}}-\sum_{I} Z_{I} \vec{R}_{I} \cdot \vec{f}^{\mathrm{ext}} \\
& -\frac{1}{2} \mathscr{M}^{\mathrm{ind}} \cdot\left(\mathscr{F}^{\mathrm{nuc}}-\mathscr{F}^{\mathrm{ele}}+\mathscr{F}^{\mathrm{ext}}\right)+E_{\mathrm{vdW}}^{\mathrm{QM} / \mathrm{MM}}
\end{aligned}
$$

will be used for the calculation of the total energy. The last term is the van der Waals (vdW) interaction between QM and MM regions. Here we adopt the distance-dependent variance of the classical Lennard-Jones (LJ) 12-6 potential according to Jensen et al. ${ }^{52}$ The expression is presented in Section S1 of the supporting information (SI).

\section{II.B. Energy Gradient and Hessian Matrix}

The first derivatives of the energy in Eq. (16) with respective to the QM coordinates, $x$, can be expressed as

$$
\begin{aligned}
E^{x}= & \mathbf{P} \cdot\left(\mathbf{h}^{[x]}+\frac{1}{2} \mathbf{I I}^{[x]} \cdot \mathbf{P}\right)+E_{\mathrm{xc}}^{[x]}-\mathbf{W} \cdot \mathbf{S}^{[x]} \\
& -\sum_{I} Z_{I} \vec{R}_{I}^{x} \cdot \vec{f}^{\mathrm{ext}}-\mathscr{M}^{\mathrm{ind}} \mathscr{F}^{\mathrm{nuc},[x]}+E_{\mathrm{vdW}}^{\mathrm{QM} / \mathrm{MM},[x]}
\end{aligned}
$$

where superscripts with [] refer to the explicit derivatives excluding the contributions from orbital rotations. Here

$$
\mathbf{h}^{[x]}=\mathbf{h}_{0}^{[x]}+\overrightarrow{\mathbf{M}}^{[x]} \cdot \vec{f}^{\mathrm{ext}}+\mathscr{M}^{\text {ind }} \mathbf{V}^{[x]}
$$

with $V_{\mu \nu}^{[x]}=\left\langle\phi_{\mu}|\mathscr{T}| \phi_{v}\right\rangle^{[x]}$. The energy weighted density matrix is

$$
\mathbf{W}=\frac{1}{2} \mathbf{P F C C}^{\dagger}+\frac{1}{2} \mathbf{C C}^{\dagger} \mathbf{F P}=\mathbf{P F P}
$$

The contributions from MM part could be found by differentiating the last two terms in Eq. (10) as

$$
E^{\mathrm{MM}, x}=-\mathscr{M}^{\mathrm{ind}} \cdot \mathscr{F}^{\mathrm{QM},[x]}+E_{\mathrm{vdW}}^{\mathrm{QM} / \mathrm{MM},[x]}
$$

where

$$
\mathscr{F} \mathrm{QM},[x]=-\mathbf{V}^{[x]} \mathbf{P}-\mathbf{V} \mathbf{P}^{[x]}+\sum_{I} Z_{I} \mathscr{T}_{I}^{[x]}
$$

derived from Eq. (6). 
The Hessian can then be obtained by directly differentiating the QM gradient in Eq. (17) with respect to the QM coordinates,

$$
\begin{aligned}
E^{x y}= & \mathbf{P} \cdot\left(\mathbf{h}^{[x y]}+\frac{1}{2} \mathbf{I I}^{[x y]} \cdot \mathbf{P}\right)+\mathbf{P}^{y} \cdot\left(\mathbf{h}^{[x]}+\mathbf{I I}^{[x]} \cdot \mathbf{P}+\mathbf{F}_{\mathrm{xc}}^{[x]}\right) \\
& +E_{\mathrm{xc}}^{[x y]}-\mathbf{W} \cdot \mathbf{S}^{[x y]}-\mathbf{W}^{y} \cdot \mathbf{S}^{[x]}-\mathscr{M}^{\mathrm{ind}} \cdot \mathscr{F}^{\mathrm{nuc},[x y]} \\
& -\mathscr{F}^{\mathrm{QM}, y} \cdot \mathscr{T}^{-1} \cdot \mathscr{F} \mathrm{QM},[x]+E_{\mathrm{vdW}}^{\mathrm{QM} / \mathrm{MM},[x y]}
\end{aligned}
$$

where

$$
\mathbf{h}^{[x y]}=\mathbf{h}_{0}^{[x y]}+\overrightarrow{\mathbf{M}}^{[x y]} \vec{f}^{\text {ext }}+\mathscr{M}^{\text {ind }} \cdot \mathbf{V}^{[x y]}
$$

with $V_{\mu v}^{[x y]}=\left\langle\phi_{\mu}|\mathscr{T}| \phi_{v}\right\rangle^{[x y]}$. The MM contributions to the Hessian matrix could be summarized as

$$
\begin{aligned}
E^{\mathrm{MM}, x y}= & -\mathscr{M}^{\mathrm{ind}} \cdot\left(\mathscr{F}^{\mathrm{QM},[x y]}-\mathbf{V}^{[x]} \mathbf{P}^{y}\right) \\
& -\mathscr{F}^{\mathrm{QM}, y} \cdot \mathscr{T}^{-1} \cdot \mathscr{F}^{\mathrm{QM},[x]}+E_{\mathrm{vdW}}^{\mathrm{QM} / \mathrm{MM},[x y]} .
\end{aligned}
$$

Here $\mathbf{P}^{y}$ includes the derivatives of orbital rotations. The first term represents the MM charges and dipoles induced by the seond-order QM potentials and fields with respect to QM nuclear coordinate changes, while the second term is the interaction between first-order QM potentials and fields and MM induced charges and dipoles from another first-order QM potentials and fields perturbed by QM atom displacements. After the construction of the Hessian matrix in the mass-weighted coordinates, and projecting the rotational and translational degrees of freedom out of this harmonic force constant matrix, one could obtain the normal modes and vibrational frequencies by diagonalizing the projected matrix. ${ }^{70}$ Note that here the Hessian matrix only includes the QM-QM block, and hence, it is actually partial Hessian within the QM region.

\section{II.C. Infrared and Raman Spectral Intensities}

IR and Raman spectra provide information about the molecular vibrations. IR spectra can be obtained from light absorption whereas Raman spectra reflect light scattering process. The IR and Raman spectral intensities are proportional to the squares of nuclear derivatives of molecular electric dipole and polarizability, respectively. ${ }^{65,70,71}$ When the perturbation is the external field $f_{m}$, the derivatives of total energy in Eq. (10) would give the total dipole moment

$$
\mu_{m}^{\mathrm{tot}}=-\frac{\partial E}{\partial f_{m}}=\sum_{I} Z_{I} R_{I, m}-\mathbf{P} \cdot \mathbf{M}_{m}+\mathscr{M}^{\mathrm{ind}} \cdot \mathscr{R}_{m}
$$

where the first two terms produce the QM dipole moment as in Eq. (12), and $\mathscr{R}_{m}$ is the $m$-th column of matrix $\mathscr{R}$ in Eq. (9). For $m=0$ (the $x$ component), for instance, $\mathscr{R}_{m}$ is $\left[R_{1 x}^{\prime}, 1,0,0, R_{2 x}^{\prime}, 1,0,0, \cdots\right]$. Then $\mathscr{M}^{\text {ind }} \mathscr{R}_{m}$ would be $\sum_{j}\left(q_{j}^{\text {ind }} R_{j m}^{\prime}+\mu_{j, m}^{\text {ind }}\right)$ with $j$ as the MM atom index.

The first-order changes of the total dipole in Eq. (25) with respect to nuclear displacements could be derived as

$$
\mu_{m}^{\mathrm{tot}, x}=\sum_{I} Z_{I} R_{I, m}^{[x]}-\mathbf{P}^{x} \cdot \mathbf{M}_{m}-\mathbf{P} \cdot \mathbf{M}_{m}^{[x]}+\mathscr{F}^{\mathrm{QM}, x} \cdot \mathscr{T}^{-1} \cdot \mathscr{R}_{m}
$$

Here the last term refers to the monopolar/dipolar response in the MM region. Namely, as one displaces the QM atoms, it leads to a variation in the corresponding potential and field $\left(\mathscr{F}^{\mathrm{QM}, x}\right)$ and thus causes a change to the induced charges and dipoles on MM atoms.

The electronic polarizability is obtained by differentiating the total dipole moment given by Eq. (25) with respect to the external field $f_{n}$,

$$
\alpha_{m n}=\frac{\partial \mu_{m}^{\mathrm{tot}}}{\partial f_{n}}=-\mathbf{P}^{n} \cdot \mathbf{M}_{m}+\left(\mathscr{F}^{\mathrm{QM}, n}+\mathscr{R}_{n}\right) \cdot \mathscr{T}^{-1} \cdot \mathscr{R}_{m}
$$

and its nuclear derivative is derived as

$$
\begin{aligned}
\alpha_{m n}^{x} & =-\mathbf{P}^{n x} \cdot \mathbf{M}_{m}-\mathbf{P}^{n} \cdot \mathbf{M}_{m}^{[x]}+\mathscr{F}^{\mathrm{QM}, n x} \cdot \mathscr{T}^{-1} \cdot \mathscr{R}_{m} \\
& =-\mathbf{P}^{n x} \cdot\left(\mathbf{M}_{m}+\mathbf{V} \mathscr{T}^{-1} \mathscr{R}_{m}\right)-\mathbf{P}^{n} \cdot\left(\mathbf{M}_{m}^{[x]}+\mathbf{V}^{[x]} \mathscr{T}^{-1} \mathscr{R}_{m}\right) \\
& =-\mathbf{P}^{n x} \cdot \tilde{\mathbf{M}}_{m}-\mathbf{P}^{n} \cdot \tilde{\mathbf{M}}_{m}^{[x]}
\end{aligned}
$$

where $\mathscr{F}^{\mathrm{QM}, n x} \cdot \mathscr{T}^{-1} \cdot \mathscr{R}_{m}$ represents the contribution of the MM part, which indicates that the two perturbations both act on the QM region (i.e., $\mathscr{F}^{\mathrm{QM}, n x}$ ) in the current framework. It is incorporated into the dipole moment matrix and its derivative matrix in the last line.

The derivatives of density matrix are derived in Appendix A. As in the case of the first-order derivatives of density matrix $\mathbf{P}^{x}$ or $\mathbf{P}^{n}$, only the first-order derivatives of orbital rotations are needed in the second-order derivatives of density matrix $\mathbf{P}^{n x}$, which is known as the $2 n+1$ rule. $^{72,73}$ The derivatives of orbital rotations are computed by the coupledperturbed Hartree-Fock equation or $z$-vector equation, which is presented in Appendix B.

\section{COMPUTATIONAL DETAILS}

The QM/DIM method and its analytic energy derivatives are implemented in a locally modified version of the Q-CHEM package. ${ }^{74}$ The parameters used in this QM/DIM method are listed in Section S1 of SI. Pyridine (Py) molecule and two gold clusters including 18 and 32 gold atoms are chosen as the test system. Based on these components, three complex configurations shown in Fig. 1 are formed: surficial $\mathrm{Py}-\mathrm{Au}_{18}$ $\mathbf{S}$, vertical $\mathrm{Py}-\mathrm{Au}_{18}-\mathbf{V}$, and $\mathrm{Py}-\mathrm{Au}_{32}$ with the nearest $\mathrm{N}-\mathrm{Au}$ distances being 3, 4, and $5 \AA$. The geometries are obtained from full QM restrained optimization with a harmonic potential, so that each of the structures has only one imaginary frequency along with the labeled $\mathrm{N}-\mathrm{Au}$ direction; their coordinates are provided in the SI. Restrained optimizations are also carried out by QM/DIM method and the obtained coordinates show no apparent difference from full QM results (with largest RMSD value as 0.007 Bohr in Table S3 of SI). The IR and Raman spectra are calculated by full QM and hybrid QM/DIM methods. In the latter, pyridine is treated by QM and the gold atoms form the DIM region. The partial Hessian is used in the QM/DIM method, whose potential errors are discussed in 
Section S3 of SI. To validate the implementation of the analytic derivatives of the QM/DIM method, analytical and finitedifferent approaches are used to compute the frequencies and Raman spectral intensities of the $\mathrm{Py}-\mathrm{Au}_{18}-\mathbf{V}$ with the labeled $\mathrm{N}$-Au distance as $3 \AA$ and the values are collected in Tab. S7 of the SI.

Furthermore, to demonstrate the capability of current analytic derivatives approaches within QM/DIM, we calculate the normal surface enhanced Raman scattering spectra (SERS) of 4,4'-bipyridine (4,4'-BPy), which is set close to the surfaces of $\mathrm{Au}_{2057}$ and $\mathrm{Ag}_{2057}$ icosahedral MNPs, respectively (their geometric arrangements are shown in Fig. S5 of SI). The simulated SERS spectra are compared with experimental ones. ${ }^{75,76}$

All the calculations are carried out with PBE0 functional and 6-31+G(d) for N, C, and $\mathrm{H}$ atoms and LanL2DZ ECP and basis set for Au atoms using the locally modified Q-CHEM 5.2 package. ${ }^{74}$ B3LYP functional is also used for $4,4^{\prime}$-BPy system for the comparison with previous theoretical works. ${ }^{77,78}$ The convergence thresholds of SCF and CPKS are both $10^{-8}$ au while a $10^{-14}$ au threshold is used for two-electron integrals. SG-1 DFT grid $^{79}$ is utilized for DFT numerical integration. The vibrational spectra are plotted using a Lorentz function with a width of $10 \mathrm{~cm}^{-1}$.

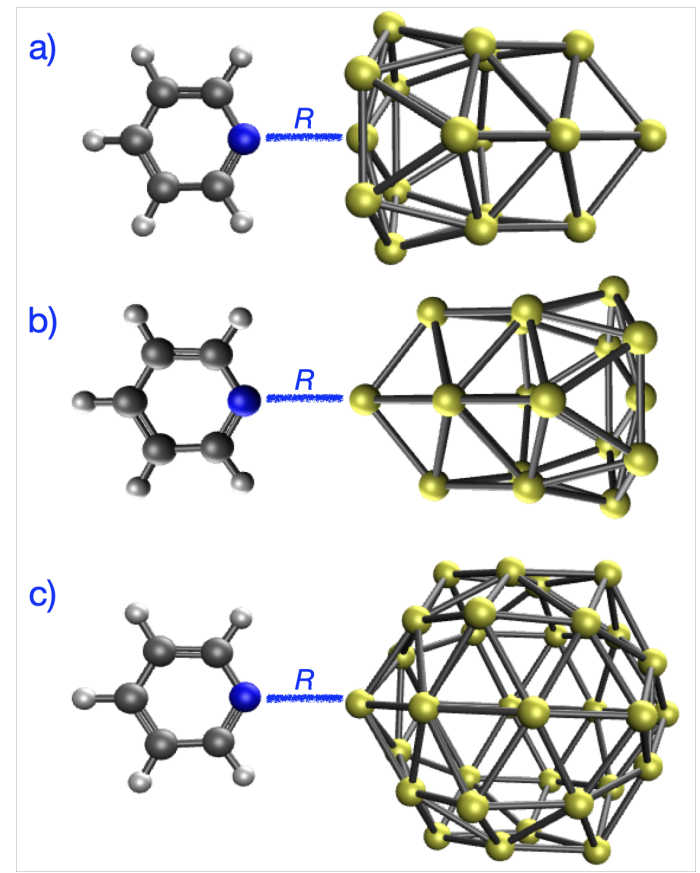

FIG. 1. Pyridine-gold cluster configurations: a) surfacial $\mathrm{Py}-\mathrm{Au}_{18}-$ $\mathbf{S}$, b) vertical $\mathrm{Py}-\mathrm{Au}_{18}-\mathbf{V}$, and c) $\mathrm{Py}-\mathrm{Au}_{32}$. The nearest N-Au distance, $R$, is set to be 3,4 , or $5 \AA$.

\section{RESULTS}

\section{IV.A. Infrared Spectra}

The infrared (IR) spectra of pyridine molecule and three different pyridine-gold complexes $\left(\mathrm{Py}-\mathrm{Au}_{18}-\mathbf{S}, \mathrm{Py}-\mathrm{Au}_{18}-\mathbf{V}\right.$, $\mathrm{Py}-\mathrm{Au}_{32}$ ) computed by QM and QM/DIM methods are displayed in Fig. 2. The top row shows the IR spectra of the gas-phase pyridine minimum-energy structure, while the other three rows show the spectra calculated using the optimized configurations (with the distances restrained at 3,4 , and $5 \AA$ ). In these three rows, the green curves correspond to the spectra of the pyridine molecule at the geometry extracted from each of these complexes. Overall, the QM/DIM method (blue curves) reproduces similar trends in the harmonic frequency shifts and comparable IR intensities relative to the full QM profiles (red curves) for the tested systems.

Overall, the harmonic vibrational frequencies of the pyridine molecule are shifted by up to $20 \mathrm{~cm}^{-1}$ from the gas phase to the complexes. Within each of the three complexes, however, the frequencies of the adsorbed pyridine molecule have marginal shifts towards higher frequency (smaller than 7 $\mathrm{cm}^{-1}$ ) upon the binding to Au clusters. This is not surprising because the heavy $\mathrm{Au}$ atoms vibrate very slowly and have a negligible effect on the nearby pyridine molecule. For a representative structure, $\mathrm{Py}-\mathrm{Au}_{18}-\mathbf{V}(3 \AA)$, the numerical values are provided in Table S13 of the SI, and information for several key vibrational modes are collected in Table I.

When the pyridine molecule approaches the Au clusters, the IR peaks of three vibrational modes (ring breathing and two $\mathrm{C}-\mathrm{H}$ wags) within $1000 \sim 1300 \mathrm{~cm}^{-1}$ become increasingly more intense as the distance reduces from 5 to $3 \AA$. The second C-H wag at $1238 \mathrm{~cm}^{-1}$ is predicted by full QM calculations to have the largest enhancement of 24 times (from 2.7 to $48.7 \mathrm{~km} / \mathrm{mol}$ ) for the $\mathbf{S}$ configuration, 33 times for $\mathbf{V}$ configuration, and 24 times for $\mathrm{Py}-\mathrm{Au}_{32}$, as shown in the panels (j), (k), and (1) of Fig. 2, respectively. In contrast, some peaks are significantly weakened after the binding to Au clusters. This is especially obvious with the two out-of-plane $\mathrm{C}-\mathrm{H}$ bends at 744 and $780 \mathrm{~cm}^{-1}$, which have comparable intensities in the gas phase. While only the IR peak at $744 \mathrm{~cm}^{-1}$ is reduced in $\mathrm{Py}-\mathrm{Au}_{18}-\mathbf{S}(3 \AA)$ and $\mathrm{Py}-\mathrm{Au}_{18}-\mathbf{V}(3 \AA)$ complexes, both peaks $\left(744 \mathrm{~cm}^{-1}\right.$ and $\left.780 \mathrm{~cm}^{-1}\right)$ lose some strength in the $\mathrm{Py}-\mathrm{Au}_{32}$ (3̊) structure.

These changes in the IR intensities are reproduced reasonably well by QM/DIM calculations, as illustrated in Fig. 2. As shown in Table I, for the $\mathrm{Py}-\mathrm{Au}_{18}-\mathrm{V}(3 \AA)$ complex, the aforementioned enhanced $\mathrm{C}-\mathrm{H}$ wag (mode 16$)$ is predicted to be $67.28 \mathrm{~km} / \mathrm{mol}$ (full QM) and $73.73 \mathrm{~km} / \mathrm{mol}$ (QM/DIM). Similarly, the calculated IR intensity of mode 14 is predicted to be enhanced to 35.86 and $38.42 \mathrm{~km} / \mathrm{mol}$, respectively, by the full-QM and QM/DIM calculations. The largest deviation appears for mode 22: the full QM method predicts an intensity of $43.62 \mathrm{~km} / \mathrm{mol}$, whereas a larger value $(66.96 \mathrm{~km} / \mathrm{mol})$ is predicted by QM/DIM. 

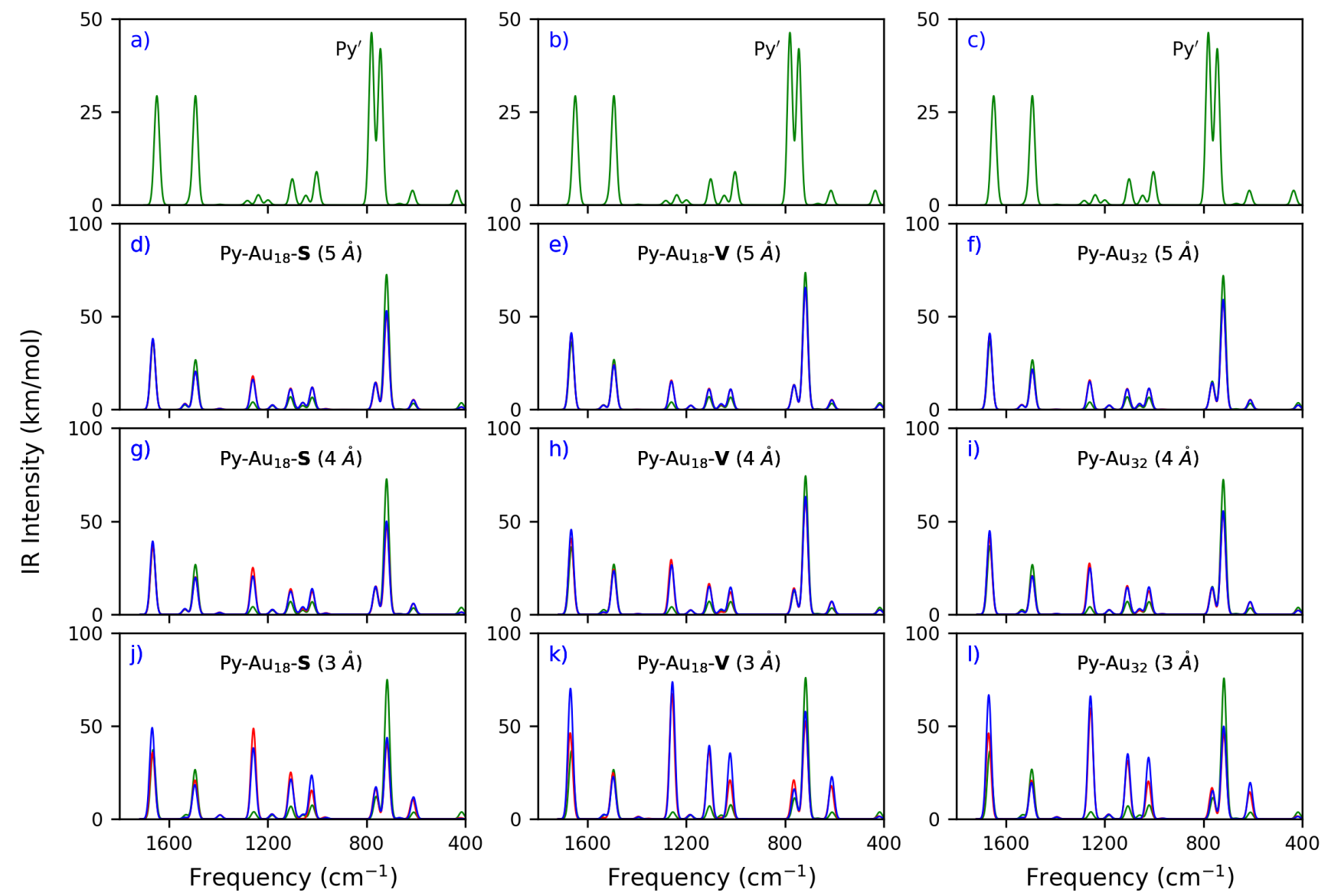

FIG. 2. Infrared spectra of the gas-phase pyridine $\left(\mathrm{Py}^{\prime}\right)$ and the $\mathrm{Py}-\mathrm{Au}_{18}-\mathbf{S}, \mathrm{Py}-\mathrm{Au}_{18}-\mathbf{V}$, and $\mathrm{Py}-\mathrm{Au}_{32}$ complexes with three intersystem distances, 3, 4, and $5 \AA$, calculated by full QM and QM/DIM methods with PBE0/6-31+G(d)/LanL2DZ. The green, red, and blue curves represent the results from the QM calculation for Py molecule and from full QM and QM/DIM for Py-Au complexes, respectively.

TABLE I. Selected vibrational frequencies and corresponding IR and Raman intensities of gas-phase Py ${ }^{\prime}$ and $\mathrm{Py}-\mathrm{Au}_{18}-\mathbf{V}$ ( $3 \AA$ ) complex calculated by full QM and QM/DIM methods with PBE0/6-31+G(d)/LanL2DZ. Here Py' and Py labels refer to the calculations based on the geometries of Py optimised in gas-phase and in $\mathrm{Py}-\mathrm{Au}_{18}$ complexes, respectively.

\begin{tabular}{|c|c|c|c|c|c|c|c|c|c|c|c|c|}
\hline \multirow[t]{3}{*}{ No. } & \multicolumn{4}{|c|}{ Freq $\left(\mathrm{cm}^{-1}\right)$} & \multicolumn{4}{|c|}{$\mathrm{I}_{\mathrm{IR}}(\mathrm{km} / \mathrm{mol})$} & \multicolumn{4}{|c|}{$\mathrm{I}_{\text {Raman }}\left(\AA^{4} / \mathrm{AMU}\right)$} \\
\hline & \multirow[t]{2}{*}{$\mathrm{Py}^{\prime}$} & \multirow[t]{2}{*}{ Py } & \multicolumn{2}{|c|}{$\mathrm{Py}-\mathrm{Au}_{18}$} & \multirow[t]{2}{*}{$\mathrm{Py}^{\prime}$} & \multirow[t]{2}{*}{$\mathrm{Py}$} & \multicolumn{2}{|c|}{$\mathrm{Py}-\mathrm{Au}_{18}$} & \multirow[t]{2}{*}{$\mathrm{Py}^{\prime}$} & \multirow[t]{2}{*}{$\mathrm{Py}$} & \multicolumn{2}{|c|}{$\mathrm{Py}-\mathrm{Au}_{18}$} \\
\hline & & & $\mathrm{QM}$ & QM/DIM & & & QM & QM/DIM & & & QM & QM/DIM \\
\hline 3 & 614.45 & 609.81 & 613.18 & 610.82 & 3.91 & 3.65 & 17.87 & 22.71 & 4.478 & 3.873 & 8.894 & 114.258 \\
\hline 4 & 666.96 & 668.16 & 666.11 & 667.73 & 0.38 & 0.40 & 0.04 & 0.04 & 5.734 & 5.037 & 4.339 & 6.816 \\
\hline 5 & 744.36 & 717.01 & 717.64 & 717.97 & 41.94 & 75.96 & 52.66 & 57.82 & 0.114 & 0.063 & 0.296 & 0.020 \\
\hline 6 & 780.49 & 761.66 & 764.78 & 763.01 & 46.29 & 11.29 & 20.94 & 16.09 & 0.040 & 0.094 & 0.029 & 0.025 \\
\hline 11 & 1042.75 & 1020.11 & 1022.95 & 1022.55 & 0.00 & 7.58 & 20.85 & 35.36 & 0.007 & 9.500 & 31.707 & 125.361 \\
\hline 12 & 1046.25 & 1058.45 & 1057.95 & 1058.29 & 2.56 & 2.05 & 0.68 & 0.01 & 41.408 & 54.055 & 294.444 & 236.590 \\
\hline 14 & 1101.06 & 1105.89 & 1108.21 & 1107.01 & 7.00 & 7.00 & 35.86 & 38.42 & 2.172 & 1.492 & 2.241 & 47.912 \\
\hline 16 & 1238.39 & 1254.27 & 1257.70 & 1255.92 & 2.73 & 3.72 & 67.28 & 73.73 & 7.583 & 8.893 & 9.845 & 11.281 \\
\hline 20 & 1513.58 & 1530.22 & 1534.97 & 1533.16 & 1.39 & 2.15 & 0.69 & 2.43 & 3.672 & 1.832 & 8.758 & 52.600 \\
\hline 22 & 1651.11 & 1665.77 & 1670.11 & 1668.91 & 22.34 & 24.82 & 43.62 & 66.96 & 12.857 & 13.733 & 12.854 & 58.173 \\
\hline
\end{tabular}

\section{IV.B. Raman Scattering Spectra}

Figs. 3 shows the calculated Raman spectra of $\mathrm{Py}-\mathrm{Au}_{18}-$ $\mathbf{S}, \mathrm{Py}-\mathrm{Au}_{18}-\mathbf{V}$, and $\mathrm{Py}-\mathrm{Au}_{32}$ complexes by full $\mathrm{QM}$ and
QM/DIM methods. The results are compared to those of the isolated pyridine. For all the configurations, we observe that the $\mathrm{Au}$ clusters can significantly enhance the ring breathing 

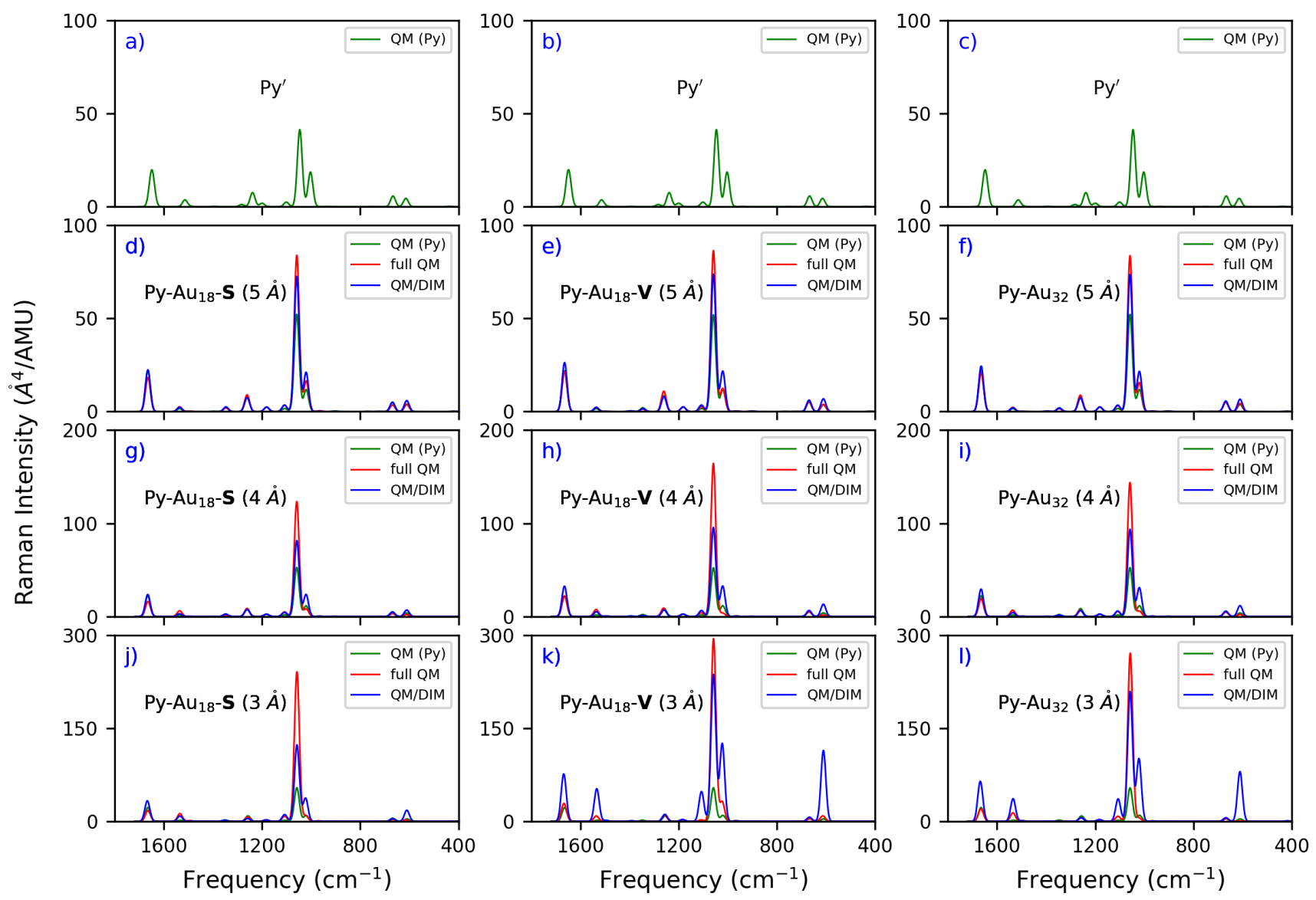

FIG. 3. Raman spectra of the gas-phase pyridine $\left(\mathrm{Py}^{\prime}\right)$ and the $\mathrm{Py}-\mathrm{Au}_{18}-\mathbf{S}, \mathrm{Py}-\mathrm{Au}_{18}-\mathbf{V}$, and $\mathrm{Py}-\mathrm{Au}_{32}$ complexes at 3,4 , and $5 \AA$ intersystem distances calculated by full QM and QM/DIM methods with PBE0/6-31+G(d)/LanL2DZ.

vibration mode 12 (around $1050 \mathrm{~cm}^{-1}$ ).

When the distance is $5 \AA$, although QM/DIM slightly underestimates the intensity of mode 12 , it nearly reproduces the full QM spectra as Figs. 3(d), 3(e), and 3(f) show. As $R$ decreases, however, QM/DIM gives less satisfying Raman profiles. Taking $\mathrm{Py}-\mathrm{Au}_{18}-\mathbf{V}$ system with $R=4 \AA$ as an example (see Fig. 3(h)), we observe that QM/DIM significantly underestimates the intensity of mode 12 but overestimates this of mode 11 compared with the full QM results. When $R$ is further shortened to $3 \AA$, the discrepancy between full QM and $\mathrm{QM} / \mathrm{DIM}$ results in all frequency ranges increases. As shown in Table I, QM/DIM significantly overestimates the intensities of all the modes except mode 12. In other words, the QM/DIM approach could provide artificial enhancements for some vibrational modes, which can be attributed to the neglect of chemical enhancement and nonlinear responses of the MNP in the current QM/DIM model.

Moreover, we calculate the normal Raman scattering spectra of 4,4'-bipyridine $\left(4,4^{\prime}\right.$-BPy) in the gas phase, and in the proximity of $\mathrm{Au}_{2057}$ and $\mathrm{Ag}_{2057}$ with respect to the DFT XC functional PBE0 and B3LYP, respectively. Fig. 4 and Fig. S6 in SI show the calculated results, where the gas-phase Raman intensities are scaled by a factor of 20 . It is noted that the spectra calculated with PBE0 and B3LYP are almost identical, indicating that the impact of functional on the spectra is small.

To compare with the experimental off-resonance SERS spectra with the incident light of $535 \mathrm{~nm}$, here the Raman scattering intensity (in unit of $\mathrm{cm}^{2} / \mathrm{sr}$ ) for the vibrational mode $k$ is calculated as

$$
I_{k}^{\text {Raman }}=\frac{(2 \pi)^{4}}{45} \frac{h}{8 \pi^{2} c \omega_{k}} \frac{\left(\omega_{0}-\omega_{k}\right)^{4}}{1-\exp \left(-h c \omega_{k} / k_{B} T\right)} S_{k}
$$

based on short-time and Placzek approximations. ${ }^{80}$ Here $\omega_{0}$ is the frequency of the incident light and $S_{k}$ (in unit of $\AA^{4} / \mathrm{AMU}$ ) refers to the Raman scattering factor,

$$
S_{k}=45 \bar{\alpha}_{k}^{\prime 2}+7 \gamma_{k}^{2}
$$

where isotropic and anisotropic polarizability derivatives, $\bar{\alpha}^{\prime}$ and $\gamma^{\prime}$, are composed by the components of nuclear derivatives 
of molecular polarizability $\alpha^{\prime}$ as,

$$
\begin{aligned}
\bar{\alpha}^{\prime}= & \frac{1}{3}\left(\alpha_{x x}^{\prime}+\alpha_{y y}^{\prime}+\alpha_{z z}^{\prime}\right) \\
\gamma^{\prime}=\frac{1}{2}( & \left(\alpha_{x x}^{\prime}-\alpha_{y y}^{\prime}\right)^{2}+\left(\alpha_{x x}^{\prime}-\alpha_{z z}^{\prime}\right)^{2}+\left(\alpha_{y y}^{\prime}-\alpha_{z z}^{\prime}\right)^{2} \\
& \left.+6\left(\alpha_{x y}^{\prime 2}+\alpha_{x z}^{\prime 2}+\alpha_{y z}^{\prime 2}\right)\right)
\end{aligned}
$$

respectively. The constants in Eq. (29) are provided in Table S2.

Fig. 4 demonstrates that, for the adsorbed configurations, QM/DIM predicts a 20 times enhancement for most of the Raman peaks due to electromagnetic enhancement. Both the theoretical calculation and experimental measurement ${ }^{75,76}$ yield a consistent result trend on the MNP-induced changes on the relative spectral intensities. For example, the gold nanoparticle makes the strongest Raman peak of $4,4^{\prime}$-BPy shift to $1678 \mathrm{~cm}^{-1}$ from $1336 \mathrm{~cm}^{-1}$, the relative intensity at around $1024 \mathrm{~cm}^{-1}$ decrease, and the two Raman peaks in the 600 $800 \mathrm{~cm}^{-1}$ range disappear. However, the QM/DIM predictions hardly alter the molecular vibrational frequencies while the experimental frequency shifts could be as large as 18 $\mathrm{cm}^{-1} .{ }^{75,76}$ It is unsurprising because after the $4,4^{\prime}$-BPy is adsorbed on the MNP, the QM/DIM optimization yields a similar geometry as the gas-phase one, thus producing only small changes in the vibrational frequencies. This is in line with the Raman spectra of the Py cases. As shown in Table I, the frequencies calculated by full QM and QM/DIM methods have similar values. The large frequency changes after molecule adsorbed on MNP come from geometry differences between Py gas-phase minimum and full QM optimized $\mathrm{Py}-\mathrm{Au}_{18} \mathrm{com}$ plex.

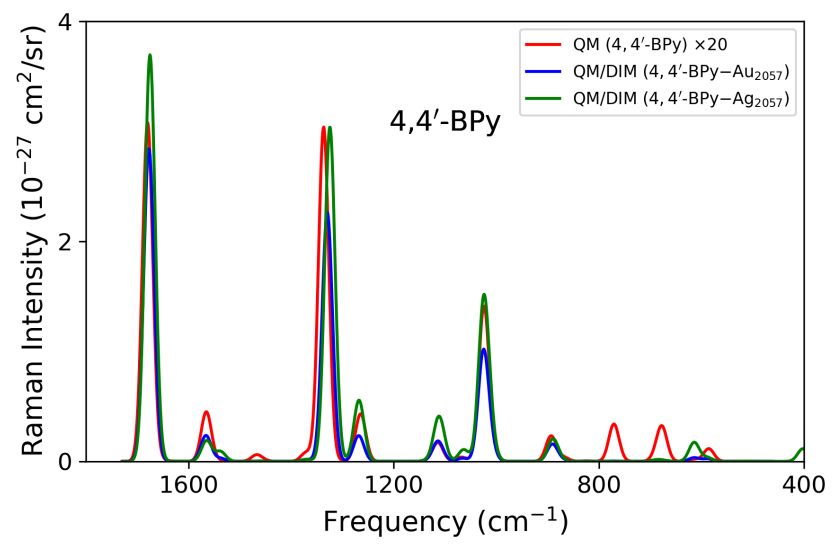

FIG. 4. Raman spectra of the gas-phase 4,4'-bipyridine (4,4'-BPy), 4,4'-BPy- $\mathrm{Au}_{2057}$ and 4,4'-BPy-Ag 2057 complexes calculated by QM and QM/DIM methods with PBE0/6-31+G(d), where QM peaks are scaled by 20 for comparison. The nearest $\mathrm{N}-\mathrm{Au}$ and $\mathrm{N}-\mathrm{Ag}$ bond lengthes are 3.034 and $2.996 \AA$, respectively.

\section{IV.C. Contribution of Nonlinear Response of MNP to Raman Intensity}

To shed light on the issue of the QM/DIM approach in the Raman calculations, we calculate the nuclear derivatives of the polarizability through the finite-difference (FD) method based on classical turning points (CTP). Two vibrational modes are chosen: the ring breathing modes around 1020 and $1050 \mathrm{~cm}^{-1}$ (modes 11 and 12). As shown in Fig. 5, the pyridine nitrogen atom shows little participation in the vibrational pattern of mode 12 while it gets involved in that of mode 11 (the same for other artificially enhanced modes).

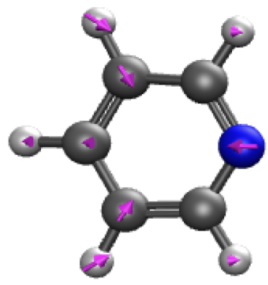

a) $\nu_{11}$

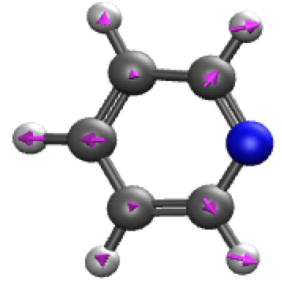

b) $\nu_{12}$
FIG. 5. Vibrational patterns of selected modes of pyridine.

As Eq. (27) shows, the molecular electric polarizability $\alpha_{m n}$ is related to the density matrix derivatives with respect to the external field and molecular dipole moment matrix. We can divide the value of $\alpha_{m n}$ into four terms with respect to the atomic basis sets contributed by its two components: the Py molecule and Au cluster. As shown in Table II, for mode 11, the Au cluster contributes 8.877 au to the final polarizability change, which largely cancels the other contributions; while the value $(-0.519 \mathrm{au})$ is negligible for mode 12 . This distinction could be understood from the vibrational motions of the pyridine. The nitrogen atom plays a major role in the vibration of the ring breathing mode 11 in Fig. 5(a) and changes the local fields exerted on the Au clusters, which requires the contribution from high-order nonlinear response terms, i.e., the hyperpolarizability-related terms of the Au atoms, to be incorporated. On the other hand, as the vibration of ring stretch mode 12 in Fig. 5(b) is symmetric along the chosen $z$ axis, there is no change in the polarizability of the Au cluster. Furthermore, there is no explicit contribution from the metal atoms to the Raman intensities in the current QM/DIM method, which can be revealed by comparing Eqs. (26) and (28).

As stated for Eq. (28), in the current QM/DIM scheme, the contribution of the MM region only comes from the induced charges and dipoles by the second-order potentials and fields of the QM counterpart, where the induced dipole moments are proportional to the first power of the field intensity. MNP may possess a strong nonlinear response, and the induced dipole moments should include the nonlinear terms that are proportional to the second and higher powers of the field intensity. Therefore, it is reasonable to account for the nonlinear response of MNPs.

The incorporation of the hyperpolarizability has been proposed by Andrews et al in their analysis of molecular Ra- 
TABLE II. Finite-difference polarizability derivatives (au) for two vibrational modes $\left(1020.11\right.$ and $\left.1058.45 \mathrm{~cm}^{-1}\right)$ of Py and Py-Au $_{18}-\mathbf{V}$ configuration at $3 \AA$ intermolecular distance calculated by full QM method with PBE0/6-31+G(d)/LanL2DZ.

\begin{tabular}{|c|c|c|c|c|c|c|c|c|c|c|}
\hline & & \multicolumn{4}{|c|}{$\mathbf{P}^{z} \cdot \Delta \mathbf{M}_{z}$} & \multicolumn{4}{|c|}{$\Delta \mathbf{P}^{z} \cdot \mathbf{M}_{z}$} & \multirow[t]{2}{*}{$\Delta \alpha_{z z}$} \\
\hline & & $\mathrm{Py}$ & $\mathrm{Py}-\mathrm{Au}$ & $\mathrm{Au}-\mathrm{Py}$ & $\mathrm{Au}$ & $\mathrm{Py}$ & $\mathrm{Py}-\mathrm{Au}$ & $\mathrm{Au}-\mathrm{Py}$ & $\mathrm{Au}$ & \\
\hline \multirow[t]{2}{*}{11} & gas-phase & 12.556 & & & & -9.862 & & & & 2.694 \\
\hline & full QM & 8.244 & -6.455 & -6.455 & 0.000 & -22.239 & 6.047 & 6.047 & 8.877 & -5.932 \\
\hline \multirow{2}{*}{12} & gas-phase & 1.419 & & & & -6.481 & & & & -5.062 \\
\hline & full QM & -5.332 & -2.057 & -2.057 & 0.000 & -32.657 & 12.617 & 12.617 & -0.519 & -17.390 \\
\hline
\end{tabular}

man scattering mediated by another molecule. ${ }^{81,82}$ The corresponding formula in their derivations is ${ }^{81}$

$$
\alpha_{m n}^{x}=\sum_{k l} R_{\mathrm{AB}}^{-3}\left(\delta_{k l}-3 \hat{e}_{k} \hat{e}_{l}\right) \mu_{l}^{\mathrm{A}, x} \beta_{m n k}^{\mathrm{B}}
$$

where $R_{\mathrm{AB}}$ is the inter-distance between two fragments, $\mathrm{A}$ and $\mathrm{B} ; \hat{e}_{k}$ is a unit number at $k$-th direction; $\vec{\mu}^{\mathrm{A}, x}$ is the nuclear derivatives of the ground state dipole moment of $\mathrm{A}$; and $\boldsymbol{\beta}^{\mathrm{B}}$ represents the electric hyperpolarizability tensor of B. It corresponds to the event that the incident and scattered photons of the Raman scattering are on the same fragment A, meanwhile one virtual photon is exchanged between the two fragments, i.e., excitation energy transfer (EET) occurs between two locally excited states of the two molecules. We note that to arrive at the hyperpolarizability-dipole interaction expression in Eq. (32), two approximations are made for the coupling between the two locally excited states: the exchange integrals are ignored and a multipole expansion of the Coulomb interaction operator is adopted. (The third one is that charge-transfer excitations are not into consideration.) This expression, however, is incomplete because the distance could also be differentiable rather than taking the first-order term in dipole moment (shown in Eq. (37)).

On other hand, if the hyperpolarizability $\boldsymbol{\beta}^{\mathrm{MM}}$ is introduced in the QM/DIM at the beginning, the additional term of the energy would be

$$
E=\frac{1}{2} \sum_{m n l} \beta_{m n l}^{\mathrm{MM}}\left(f_{m}^{\mathrm{QM}}+f_{m}^{\mathrm{ext}}\right)\left(f_{n}^{\mathrm{QM}}+f_{n}^{\mathrm{ext}}\right)\left(f_{l}^{\mathrm{QM}}+f_{l}^{\mathrm{ext}}\right)
$$

whose second-order derivative with respect to external field is

$$
\begin{array}{r}
\alpha_{i j}=\frac{1}{2} \sum_{m n l} \beta_{m n l}^{\mathrm{MM}}\left[3 f_{m}^{\mathrm{QM}, i j}\left(f_{n}^{\mathrm{QM}}+f_{n}^{\mathrm{ext}}\right)\left(f_{l}^{\mathrm{QM}}+f_{l}^{\mathrm{ext}}\right)\right. \\
\left.+6\left(f_{m}^{\mathrm{QM}, i}+\delta_{i m}\right)\left(f_{n}^{\mathrm{QM}, j}+\delta_{j n}\right)\left(f_{l}^{\mathrm{QM}}+f_{l}^{\mathrm{ext}}\right)\right]
\end{array}
$$

Then its nuclear derivative is derived as

$$
\begin{gathered}
\alpha_{i j}^{x}=\sum_{m n l} 3 \beta_{m n l}^{\mathrm{MM}}\left(f_{m}^{\mathrm{QM}, i}+\delta_{i m}\right)\left(f_{n}^{\mathrm{QM}, j}+\delta_{j n}\right) f_{l}^{\mathrm{QM}, x} \\
+\sum_{m n l} \beta_{m n l}^{\mathrm{MM}} f_{l}^{\mathrm{QM}}\left[6 f_{m}^{\mathrm{QM}, i x}\left(f_{n}^{\mathrm{QM}, j}+\delta_{j n}\right)+3 f_{m}^{\mathrm{QM}, i j} f_{n}^{\mathrm{QM}, x}\right. \\
\left.+\frac{3}{2} f_{m}^{\mathrm{QM}, i j x} f_{n}^{\mathrm{QM}}\right]
\end{gathered}
$$

Here we had assumed $f^{\text {ext }}=0$ as in the case of no external field. The first line at the right-hand-side in the above expression closely resembles Eq. (32): Provided that the electric field is generated by a dipole as $f_{l}^{\mathrm{QM}}=R_{\mathrm{AB}}^{-3}\left(\delta_{k l}-3 \hat{e}_{k} \hat{e}_{l}\right) \mu_{l}^{\mathrm{A}}$, its nuclear derivative would be

$$
f_{l}^{\mathrm{QM}, x}=R_{\mathrm{AB}}^{-3}\left(\delta_{k l}-3 \hat{e}_{k} \hat{e}_{l}\right) \mu_{l}^{\mathrm{A}, x}+\left(R_{\mathrm{AB}}^{-3}\left(\delta_{k l}-3 \hat{e}_{k} \hat{e}_{l}\right)\right)^{x} \mu_{l}^{\mathrm{A}}
$$

Then the interaction of the first term and the hyperpolarizability yeilds Eq. (32), while the second term is from the derivatives of the distance rather than molecular dipole. The rest contributions to the nuclear derivatives of polarizability $\alpha_{i j}^{x}$ in Eq. (36) includes the expression $\beta_{m n l}^{\mathrm{MM}} f_{l}^{\mathrm{QM}}$, referring to the first-order change in metal's polarizability induced by QM field. As in the QM/DIM method, each metal atom is induced by QM potential and field by atomic capacitance and polarizability and the induced charges and dipoles interact with each other, it wold be difficult to distinguish this mutual polarization from the contributions in Eq. (36) with the use of molecular hyperpolarizability parameter for metal cluster. Therefore, we use Eq. (35) to account the effect of hyperpolarizability of metal in the following.

TABLE III. Hyperpolarizability (au) of the $\mathrm{Au}_{18}$ cluster calculated using finite-difference with a field strength of 0.001 au at the PBE0/LanL2DZ level. Coordinates are obtained from the restrained optimization of $\mathrm{Py}-\mathrm{Au}_{18}-\mathrm{V}(3 \AA)$.

\begin{tabular}{rrrrr}
\hline \hline \multicolumn{1}{c}{$x$} & \multicolumn{1}{c}{$x$} & \multicolumn{1}{c}{$y$} \\
\hline & $x$ & 3.979 & 0.218 & -338.778 \\
& $y$ & 0.218 & 3.168 & -20.482 \\
& $z$ & -338.778 & -20.482 & -10.166 \\
& $x$ & 0.218 & 3.168 & -20.482 \\
& $y$ & 3.168 & 0.944 & -792.040 \\
& $z$ & -20.482 & -792.040 & 0.337 \\
$z$ & $x$ & -338.778 & -20.482 & -10.166 \\
& $y$ & -20.482 & -792.040 & 0.337 \\
& $z$ & -10.166 & 0.337 & 1929.883 \\
\hline \hline
\end{tabular}

This correction is sufficient for our purpose because the gold cluster has large hyperpolarizability and it has little participation in the vibrational modes of the Raman spectral region we are interested in. The hyperpolarizability of the $\mathrm{Au}_{18}$ cluster calculated by the finite-difference method with a field strength of 0.001 au is given in Table III. Consistent values are 
obtained with a smaller field strength (0.0002 au). As demonstrated in Table III, the calculated hyperpolarizability of the $\mathrm{Au}_{18}$ cluster has large components along the $\mathrm{z}$ axis, comparable with the values reported in the literature. ${ }^{83,84}$

The corrected QM/DIM Raman intensities of selected modes of $\mathrm{Py}-\mathrm{Au}_{18}-\mathbf{V}(3 \AA)$ complex is shown in Table IV. There are five variations $(a-e)$ of the hyperpolarizabilityrelated correction for the QM/DIM method since some arbitrariness arises when only the molecular hyperpolarizability parameter is available rather than the atomic ones. The $(a)$, (b), and (c) columns are calculated by taking the distance $R_{\mathrm{AB}}$ in Eq. (32) as the distance between the two centers of mass $(\mathrm{COM})$, the COM of pyridine and the location of the nearest gold atom, and the shortest distance of the two fragments, respectively. In other words, the distance $R_{\mathrm{AB}}$ reduces gradually when moving from $(a)$ to $(c)$. The $(d)$ and $(e)$ columns collect the Raman intensities corrected using Eq. (35) without and with QM induced fields, respectively. Even though the QM/DIM Raman intensity of mode 11 is still larger than the full QM one, it is unsurprisingly reduced with the correction of the hyperpolarizability dependent term. On the other hand, the intensity of mode 12 is also slightly increased by the $(a)$, (b), and (c) corrections.

TABLE IV. Corrected Raman intensities ( $\left.\AA^{4} / \mathrm{AMU}\right)$ by QM/DIM and reference intensities by full QM for vibrational modes 11 and 12 of $\mathrm{Py}-\mathrm{Au}_{18}-\mathbf{V}(3 \AA)$ complex with PBE0/6-31+G(d)/LanL2DZ. $(a-c)$ variations of QM/DIM method use Eq. (32) with different distance $R_{\mathrm{AB}}$ while $(d)$ and (e) correspond to Eq. (35) without and with $\mathrm{QM}$ induced fields, respectively.

\begin{tabular}{|c|c|c|c|c|c|c|}
\hline \multirow[t]{2}{*}{ No. } & \multicolumn{5}{|c|}{$\begin{array}{l}\mathrm{I} \\
\mathrm{IMaman}\end{array}$} & \multirow[t]{2}{*}{$\mathrm{I}_{\text {Raman }}^{\mathrm{QM}}$} \\
\hline & 0 & $a$ & $b$ & $c$ & $d$ & \\
\hline
\end{tabular}

$\begin{array}{llllllll}11 & 125.361 & 122.367 & 103.807 & 78.106 & 102.574 & 65.860 & 31.707\end{array}$ $12 \quad 236.590236 .679237 .306238 .874211 .852 \quad 168.580294 .444$

Fig. 6 displays the corrected QM/DIM Raman spectrum of the $\mathrm{Py}-\mathrm{Au}_{18}-\mathbf{V}(3 \AA)$ complex. For approach $(c)$, even though more correct intensity is reproduced for mode 11, several modes are wrongly enhanced such as 5, 16, and 19 . It reflects that the distance is too small within this calculation. When the distance is larger, for instance, in the case of approach $(b)$, the green curve moderately approaches the QM one in Fig. 6. On the other hand, the $(e)$ profile (blue line) improves the intensities of almost all the vibrational modes except that the intensity of mode 16 is overestimated and that of mode 12 is further reduced relative to the uncorrected QM/DIM result (from 236 to $168 \AA^{4} / \mathrm{AMU}$, while the full QM reference is 294 $\AA^{4}$ /AMU). It is acceptable though that these corrections could not produce overall satisfying intensities for all the modes, because only the molecular hyperpolarizability of the gold cluster is employed in these calculations rather than the distributed atomic hyperpolarizabilities while the fields and their derivatives act on individual gold atoms.

\section{IV.D. Population Analysis}

Raman signals can be enhanced by the chemical enhancement and the electromagnetic enhancement. In the current QM/DIM scheme, we don't account for the intermolecular charge transfer (CT) effect, which may be partially responsible for the limited accuracy of the QM/DIM scheme for Raman spectral calculations, especially when the intermolecular distance is short. To check this effect, we calculated the amount of charges on pyridine molecule within the complexes as shown in Table V.

Among the four used population methods, only the electrostatic potential (ESP) and fragment-based Hirshfeld (FBH) charge schemes consistently predict the expected trend in the fragment charge population, namely an enhanced CT with a shorter distance between two fragments. These values clearly show that the net transferred charge between Py and Au cluster is almost zero when the nearest distance between these two fragments is $5 \AA$, while it can be as large as $0.16 \mathrm{e}^{-}$when the distance shortens to $3 \AA$ in $\mathrm{Py}-\mathrm{Au}_{18}-\mathrm{V}$ according to the $\mathrm{FBH}$ calculation. It suggests that the ground state charge migration between the Py molecule and Au cluster might also play an important role in the limited accuracy of our current QM/DIM Raman spectral simulations.

\section{CONCLUDING REMARKS}

In this work, we derived and implemented the QM/DIM method and its high-order energy derivatives for the calculation of noble MNP-mediated molecular vibrational spectra, including IR and Raman scattering. Taking the complexes composed of a pyridine molecule with MNPs consisting of 18 or 32 gold atoms as testing examples, we assessed the accuracy of the current QM/DIM scheme in the calculations of molecular vibrational frequencies and IR/Raman intensities. The QM/DIM method demonstrates the ability to reproduce the molecular vibrational frequencies and IR spectral intensities obtained using the full QM approach. The Raman profiles from QM/DIM calculations behave well for large intermolecular distances such as $5 \AA$ for the tested complexes, while it shows artificial enhancements at shorter molecule-MNP distances for some of the vibrational modes. Even though, with large MNPs such as $\mathrm{Au}_{2057}$ and $\mathrm{Ag}_{2057}$, the simulated normal SERS of $4,4^{\prime}$-BPy by QM/DIM method are comparable with experimental spectra.

The deviation of QM/DIM results from full QM results for small metal clusters, however, can be partially resolved. By incorporating the term that incorporates the first-order nonlinear polarizability of Au clusters and the dipole (or electric field) derivatives of the probe molecule, we can capture the non-negligible three-virtual-photon process of Raman scattering within this hybrid method. With this correction, the Raman spectra for the short-distance configurations of the Py$\mathrm{Au}$ clusters calculated using QM/DIM are improved. We thus suggest that the atomic (distributed) hyperpolarizability of metal atoms should be parameterized within the hybrid QM/DIM method to enable it to describe Raman scat- 


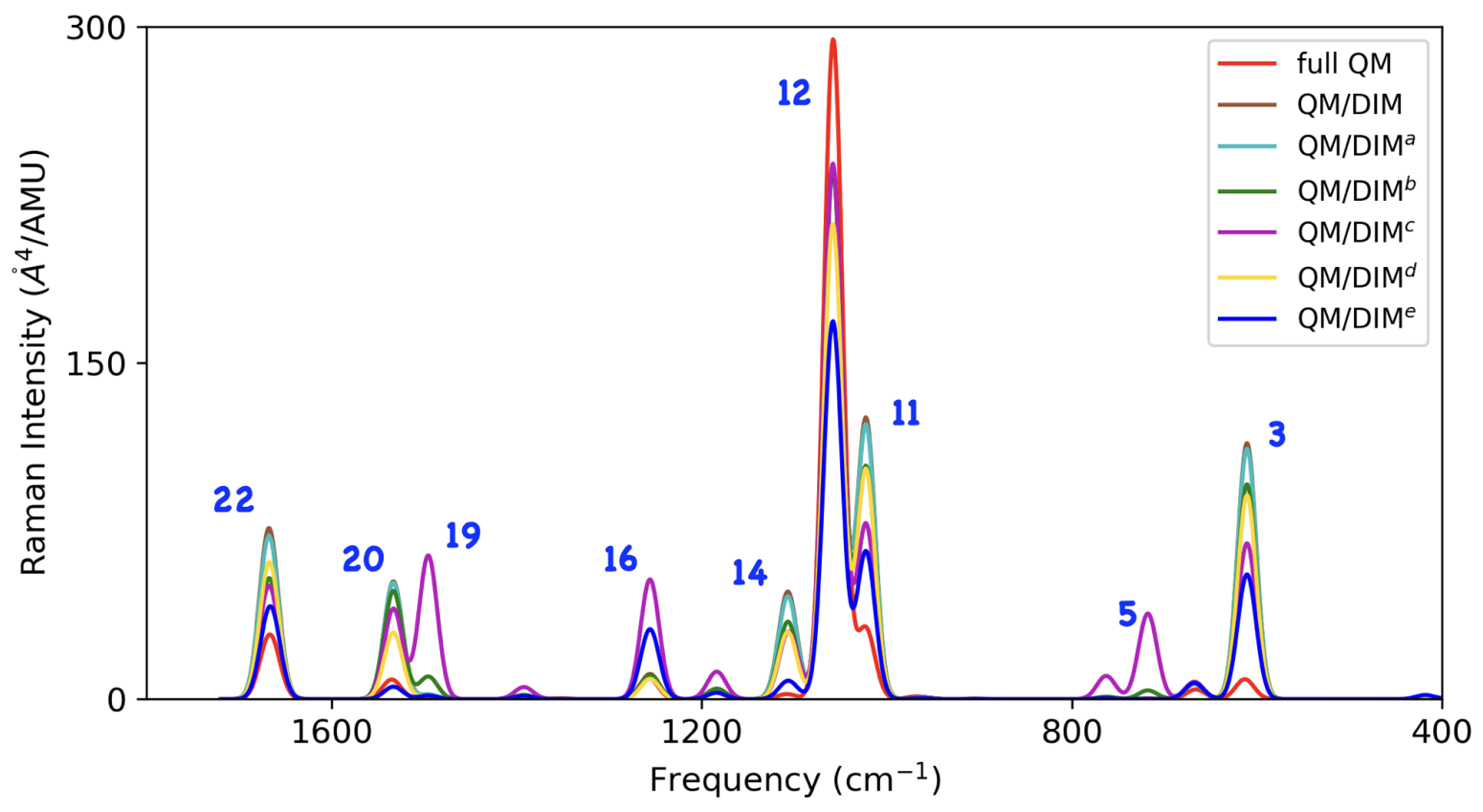

FIG. 6. Corrected Raman spectrum of Py-Au $18-\mathbf{V}(3 \AA$ A) complex by QM/DIM with PBE0/6-31+G(d)/LanL2DZ. (a-c) variations of QM/DIM method use Eq. (32) with different distance $R_{\mathrm{AB}}$ while $(d)$ and $(e)$ correspond to Eq. (35) without and with QM induced fields, respectively.

TABLE V. Charge populations on the pyridine molecule within the different Py-Au complexes calculated from the Mulliken, ${ }^{85} \mathrm{ChElPG},{ }^{86}$ $\mathrm{ESP}^{87}$ and $\mathrm{FBH}^{88}$ population schemes with PBE0/6-31+G(d)/LanL2DZ.

\begin{tabular}{|c|c|c|c|c|c|c|c|c|c|}
\hline & \multicolumn{3}{|c|}{$\mathrm{Py}-\mathrm{Au}_{18}-\mathbf{S}$} & \multicolumn{3}{|c|}{$\mathrm{Py}-\mathrm{Au}_{18}-\mathbf{V}$} & \multicolumn{3}{|c|}{$\mathrm{Py}-\mathrm{Au}_{32}$} \\
\hline & $5 \AA$ & $4 \AA$ & $3 \AA$ & $5 \AA$ & $4 \AA$ & $3 \AA$ & $5 \AA$ & $4 \AA$ & $3 \AA$ \\
\hline Mulliken & -0.12 & -0.23 & -0.01 & -0.01 & -0.02 & 0.12 & 0.00 & 0.00 & 0.09 \\
\hline ChElPG & -0.06 & -0.09 & -0.05 & -0.03 & -0.01 & 0.09 & -0.05 & -0.04 & 0.01 \\
\hline ESP & 0.00 & 0.00 & 0.02 & 0.01 & 0.04 & 0.16 & 0.00 & 0.02 & 0.10 \\
\hline FBH & 0.00 & 0.01 & 0.05 & 0.00 & 0.02 & 0.10 & 0.00 & 0.02 & 0.08 \\
\hline
\end{tabular}

tering spectra more accurately. Besides, as investigated by Schatz and the coworker, ${ }^{89}$ the other high-order properties such as dipole-quadrupole and dipole-magnetic-dipole polarizabilities may also play a rule in the Raman intensity if the electric field derivatives are comparable with the field strength.

Furthermore, at short intermolecular distances, the overlap of wave functions opens the possibility of a charge migration between Py and the Au cluster. In those cases, the effect of intermolecular CT on the Raman signal should not be neglected, which may otherwise limit the applicability of the current QM/DIM scheme for Raman spectral simulations. It is therefore desirable to develop a scheme to account for the $\mathrm{CT}$ effect in future QM/MM methods. The use of only QMQM block Hessian matrix in the current work is also a potential source of small errors in the simulated vibrational spectra. In addition, the vdW interaction between the molecule and MNP could affect the optimized molecular structure and then its vibrational frequencies predicted by using the QM/DIM method.
Finally, we note that the plasmon resonance effect of MNPs has not been taken into account in this work. We expect to include the resonance effect by adopting the frequencydependent parameters for metal atoms in future publications, which requires solving complex-valued response equations.

\section{SUPPLEMENTARY MATERIAL}

Parameters used in DIM model and Raman intensity calculation; Configurations of $4,4^{\prime}$-bipyridine $\left(4,4^{\prime}-\mathrm{BPy}\right)$ and $4,4^{\prime}$ $\mathrm{BPy}-\mathrm{Au}_{2057}$ and 4,4'-BPy-Ag 2057 complexes; numerical comparison between finite-difference and analytical results of vibrational frequencies and Raman intensities for $\mathrm{Py}-\mathrm{Au}_{18}-\mathbf{V}$ (3 $\AA$ ) configuration calculated by QM/DIM method; numerical results of frequencies and IR and Raman intensities of PyAu cluster, 4,4'-BPy-Au 2057 , and 4,4'-BPy-Ag 2057 complexes; and optimized geometry coordinates of studied $\mathrm{Py}-\mathrm{Au}$ cluster complexes. 


\section{ACKNOWLEDGMENTS}

WL acknowledges financial support from the National Natural Science Foundation of China (Grant Nos. 21833006 and 22173074). YS is supported by the National Institutes of Health (grant: R01GM135392), National Science Foundation (CHE-2102071), and the Office of the Vice President of Research and the College of Art and Sciences at the University of Oklahoma (OU).

\section{DATA AVAILABILITY STATEMENT}

The data that support the findings of this study are available within the article and its supplementary material.

\section{Appendix A: Derivatives of Density Matrix}

The occupied MO coefficients could be expanded to the second-order in orbital rotations $\Theta_{\mathrm{vo}}$ as Ref. 90 suggested.

$$
\mathbf{C}_{\mathrm{o}} \rightarrow \mathbf{C}_{\mathrm{o}}+\mathbf{C}_{\mathrm{v}} \Theta_{\mathrm{vo}}-\frac{1}{2} \mathbf{C}_{\mathrm{o}} \Theta_{\mathrm{vo}}^{T} \Theta_{\mathrm{vo}}
$$

Its first-order derivative is

$$
\mathbf{C}_{\mathrm{o}}^{x} \rightarrow \mathbf{C}_{\mathrm{o}}^{[x]}+\mathbf{C}_{\mathrm{v}}^{[x]} \Theta_{\mathrm{vo}}+\mathbf{C}_{\mathrm{v}} \Theta_{\mathrm{vo}}^{[x]} \rightarrow \mathbf{C}_{\mathrm{o}}^{[x]}+\mathbf{C}_{\mathrm{v}} \Theta_{\mathrm{vo}}^{[x]}
$$

without second-order terms while the second-order derivative could be further written as

$$
\begin{aligned}
\mathbf{C}_{\mathrm{o}}^{x y} \rightarrow & \mathbf{C}_{\mathrm{o}}^{[x y]}+\mathbf{C}_{\mathrm{v}}^{[x]} \Theta_{\mathrm{vo}}^{[y]}+\mathbf{C}_{\mathrm{v}}^{[y]} \Theta_{\mathrm{vo}}^{[x]}+\mathbf{C}_{\mathrm{v}} \Theta_{\mathrm{vo}}^{[x y]} \\
& -\frac{1}{2}\left(\mathbf{C}_{\mathrm{o}} \Theta_{\mathrm{vo}}^{[x], T} \Theta_{\mathrm{vo}}^{[y]}+\mathbf{C}_{\mathrm{o}} \Theta_{\mathrm{vo}}^{[y], T} \Theta_{\mathrm{vo}}^{[x]}\right)
\end{aligned}
$$

where we set $\Theta_{\text {vo }}=0$ as the MOs are optimized. Here

$$
\mathbf{C}_{\mathrm{o}}^{[x]}=-\frac{1}{2} \mathbf{C C}^{T} \mathbf{S}^{[x]} \mathbf{C}_{\mathrm{o}}, \quad \mathbf{C}_{\mathrm{v}}^{[x]}=-\frac{1}{2} \mathbf{C C}^{T} \mathbf{S}^{[x]} \mathbf{C}_{\mathrm{v}}
$$

When the perturbation $(x)$ in Eq. A2 is the external field in the $n$ direction, it is simplified to

$$
\mathbf{C}_{\mathrm{o}}^{n}=\mathbf{C}_{\mathrm{v}} \Theta_{\mathrm{vo}}^{[n]}
$$

Similarly, if the second perturbation (y) in Eq. (A3) is the external field in the $n$-th direction, it reduces to

$$
\mathbf{C}_{\mathrm{o}}^{x n} \rightarrow \mathbf{C}_{\mathrm{v}}^{[x]} \Theta_{\mathrm{vO}}^{[n]}+\mathbf{C}_{\mathrm{v}} \Theta_{\mathrm{vo}}^{[x n]}-\frac{1}{2}\left(\mathbf{C}_{\mathrm{o}} \Theta_{\mathrm{vo}}^{[x], T} \Theta_{\mathrm{vo}}^{[n]}+\mathbf{C}_{\mathrm{o}} \Theta_{\mathrm{vO}}^{[n], T} \Theta_{\mathrm{vo}}^{[x]}\right)
$$

As the derivatives of density matrix are dependent on the derivatives of occupied MOs

$$
\begin{aligned}
\mathbf{P}^{x} & =\mathbf{C}_{\mathrm{o}}^{x} \mathbf{C}_{\mathrm{o}}^{T}+\mathbf{C}_{\mathrm{o}} \mathbf{C}_{\mathrm{o}}^{x, T} \\
\mathbf{P}^{x y} & =\mathbf{C}_{\mathrm{o}}^{x y} \mathbf{C}_{\mathrm{o}}^{T}+\mathbf{C}_{\mathrm{o}} \mathbf{C}_{\mathrm{o}}^{x y, T}+\mathbf{C}_{\mathrm{o}}^{x} \mathbf{C}_{\mathrm{o}}^{y, T}+\mathbf{C}_{\mathrm{o}}^{y} \mathbf{C}_{\mathrm{o}}^{x, T}
\end{aligned}
$$

we have

$$
\begin{aligned}
\mathbf{P}^{x} & =\mathbf{C}_{\mathrm{o}}^{[x]} \mathbf{C}_{\mathrm{o}}^{T}+\mathbf{C}_{\mathrm{o}} \mathbf{C}_{\mathrm{o}}^{[x], T}+\mathbf{C}_{\mathrm{v}} \Theta_{\mathrm{vo}}^{[x]} \mathbf{C}_{\mathrm{o}}^{T}+\mathbf{C}_{\mathrm{o}} \Theta_{\mathrm{vo}}^{[x], T} \mathbf{C}_{\mathrm{v}}^{T} \\
& =\mathbf{C}\left(\begin{array}{cc}
-\mathbf{S}_{\mathrm{oO}}^{[x]} & -\frac{1}{2} \mathbf{S}_{\mathrm{ov}}^{[x]}+\Theta_{\mathrm{vo}}^{[x], T} \\
-\frac{1}{2} \mathbf{S}_{\mathrm{vo}}^{[x]}+\Theta_{\mathrm{vo}}^{[x]} & 0
\end{array}\right) \mathbf{C}^{T} \\
\mathbf{P}^{n} & =\mathbf{C}\left(\begin{array}{cc}
0 & \Theta_{\mathrm{vo}}^{[n], T} \\
\Theta_{\mathrm{vo}}^{[n]} & 0
\end{array}\right) \mathbf{C}^{T}
\end{aligned}
$$

and

$$
\begin{aligned}
\mathbf{P}^{x n} & =\mathbf{C}_{\mathrm{v}}^{[x]} \Theta_{\mathrm{vo}}^{[n]} \mathbf{C}_{\mathrm{o}}^{T}+\mathbf{C}_{\mathrm{o}} \Theta_{\mathrm{vo}}^{[n], T} \mathbf{C}_{\mathrm{v}}^{[x], T}+\mathbf{C}_{\mathrm{v}} \Theta_{\mathrm{vo}}^{[x n]} \mathbf{C}_{\mathrm{o}}^{T}+\mathbf{C}_{\mathrm{o}} \Theta_{\mathrm{vo}}^{[x n], T} \mathbf{C}_{\mathrm{v}}^{T} \\
& -\mathbf{C}_{\mathrm{o}} \Theta_{\mathrm{vo}}^{[x], T} \Theta_{\mathrm{vo}}^{[n]} \mathbf{C}_{\mathrm{o}}^{T}-\mathbf{C}_{\mathrm{o}} \Theta_{\mathrm{vo}}^{[n], T} \Theta_{\mathrm{vo}}^{[x]} \mathbf{C}_{\mathrm{o}}^{T}+\mathbf{C}_{\mathrm{o}}^{[x]} \Theta_{\mathrm{vo}}^{[n], T} \mathbf{C}_{\mathrm{v}}^{T} \\
& +\mathbf{C}_{\mathrm{v}} \Theta_{\mathrm{vo}}^{[n]} \mathbf{C}_{\mathrm{o}}^{[x], T}+\mathbf{C}_{\mathrm{v}} \Theta_{\mathrm{vo}}^{[x]} \Theta_{\mathrm{vo}}^{[n], T} \mathbf{C}_{\mathrm{v}}^{T}+\mathbf{C}_{\mathrm{v}} \Theta_{\mathrm{vo}}^{[n]} \Theta_{\mathrm{vo}}^{[x], T} \mathbf{C}_{\mathrm{v}}^{T} \\
& =-\frac{1}{2}\left(\mathbf{C} \mathbf{C}^{T} \mathbf{S}^{[x]} \mathbf{P}^{n}+\mathbf{P}^{n} \mathbf{S}^{[x]} \mathbf{C} \mathbf{C}^{T}\right) \\
& +\mathbf{C}\left(\begin{array}{cc}
-\Theta_{\mathrm{vo}}^{[x], T} \Theta_{\mathrm{vo}}^{[n]}-\Theta_{\mathrm{vo}}^{[n], T} \Theta_{\mathrm{vo}}^{[x]} & \Theta_{\mathrm{vo}}^{[n x], T} \\
\Theta_{\mathrm{vo}}^{[n x]} & \Theta_{\mathrm{vo}}^{[x]} \Theta_{\mathrm{vo}}^{[n], T}+\Theta_{\mathrm{vo}}^{[n]} \Theta_{\mathrm{vo}}^{[x], T}
\end{array}\right) \mathbf{C}^{T}
\end{aligned}
$$

The derivatives of orbital rotations $\left(\Theta_{\mathrm{vo}}^{[x]}, \Theta_{\mathrm{vo}}^{[n]}\right.$, and $\left.\Theta_{\mathrm{vo}}^{[n x]}\right)$ are determined using the procedure outlined in the next section.

\section{Appendix B: Coupled-Perturbed Self-Consistent Field and $z$-Vector Equations}

To obtain the first-order changes in the density matrix, we need to solve the coupled-perturbed self-consistent field (CP$\mathrm{SCF}$ ) equation. Differentiating the SCF convergence condition $[\mathbf{F}, \mathbf{P}]_{\mathbf{S}}=\mathbf{F P S}-\mathbf{S P F}=0$ with respective to an external perturbation $x$, we have

$$
\left[\mathbf{F}^{x}, \mathbf{P}\right]_{\mathbf{S}}+\left[\mathbf{F}, \mathbf{P}^{x}\right]_{\mathbf{S}}+[\mathbf{F}, \mathbf{P}]_{\mathbf{S}^{x}}=0
$$

where $\mathbf{F}^{x}=\mathbf{F}^{[x]}+\mathbf{F}^{(2)} \cdot \mathbf{P}^{x}$. After moving the $\mathbf{P}^{x}$ dependent terms to one side, we arrive at

$$
\left[\mathbf{F}, \mathbf{P}^{x}\right]_{\mathbf{S}}+\left[\mathbf{F}^{(2)} \cdot \mathbf{P}^{x}, \mathbf{P}\right]_{\mathbf{S}}=-\left[\mathbf{F}^{[x]}, \mathbf{P}\right]_{\mathbf{S}}-[\mathbf{F}, \mathbf{P}]_{\mathbf{S}^{x}}
$$

where $\mathbf{F}^{(2)}=\mathbf{I I}+\boldsymbol{\Omega}+\mathbf{V} \mathscr{T}^{-1} \mathbf{V}$. Note that $\boldsymbol{\Omega}$ is the exchangecorrelation portion of the response kernel as defined in Eq. (14) of Ref. 91. The explicit Fock matrix derivatives, $\mathbf{F}^{[x]}=$ $\mathbf{h}^{[x]}+\mathbf{I I}^{[x]} \cdot \mathbf{P}+\mathbf{F}_{\mathrm{xc}}^{[x]}$, where $\mathbf{h}^{[x]}$ includes the contribution from the MM region as shown in Eq. (17) and $\mathbf{F}_{\mathrm{Xc}}^{[x]}$ is defined in Eq. (25) in Ref. 91. Projecting the first-order equation-of-motion in Eq. (B2) into vo block, we have

$$
(\mathbf{A}+\mathbf{B}) \Theta_{\mathrm{vo}}^{[x]}=\mathbf{L}_{\mathrm{vo}}^{[x]}
$$

where $(\mathbf{A}+\mathbf{B})$ is the orbital Hessian, including the Fock derivatives with respect to orbital rotations. The right-handside is the vo block of the Lagrangian

$$
\begin{aligned}
\mathbf{L}^{[x]}= & \mathbf{h}_{0}^{[x]}+\mathbf{I I}^{[x]} \cdot \mathbf{P}+\mathbf{F}_{\mathrm{xc}}^{[x]}+\overrightarrow{\mathbf{M}}^{[x]} \vec{f}^{\mathrm{ext}}+(\mathbf{I I}+\mathbf{\Omega}) \cdot \mathbf{P}^{[x]} \\
& -\frac{1}{2}\left(\mathbf{S}^{[x]} \mathbf{C C}^{T} \mathbf{F}+\mathbf{F} \mathbf{C} \mathbf{C}^{T} \mathbf{S}^{[x]}\right) \\
& +\mathscr{M}^{\text {ind }} \mathbf{V}^{[x]}+\mathscr{F}^{\mathrm{tot},[x]} \mathscr{T}^{-1} \mathbf{V}
\end{aligned}
$$


for QM nuclear perturbations, where the last line at the righthand-side represents the contribution from MM part. And one uses

$$
\mathbf{L}^{[n]}=\mathbf{M}_{n}+\mathscr{R}_{n} \mathscr{T}^{-1} \mathbf{V}
$$

to solve for $\Theta_{\mathrm{vo}}^{[n]}$ with external field perturbations. The products of property vectors and response properties are interchangeable, for instance, $\Theta_{\mathrm{vo}}^{[x]} \cdot \mathbf{L}_{\mathrm{vo}}^{[a]}=\mathbf{L}_{\mathrm{vo}}^{[x]} \cdot(\mathbf{A}+\mathbf{B})^{-1} \cdot \mathbf{L}_{\mathrm{vo}}^{[a]}=$ $\mathbf{L}_{\mathrm{vo}}^{[x]} \cdot \Theta_{\mathrm{vo}}^{[a]}$.

The second-order CPSCF equation derived from Eq. (B1) is

$$
\begin{aligned}
& {\left[\mathbf{F}^{n x}, \mathbf{P}\right]_{\mathbf{S}}+\left[\mathbf{F}^{n}, \mathbf{P}^{x}\right]_{\mathbf{S}}+\left[\mathbf{F}^{x}, \mathbf{P}^{n}\right]_{\mathbf{S}}+\left[\mathbf{F}, \mathbf{P}^{n x}\right]_{\mathbf{S}}} \\
& +\left[\mathbf{F}^{n}, \mathbf{P}\right]_{\mathbf{S}^{x}}+\left[\mathbf{F}, \mathbf{P}^{n}\right]_{\mathbf{S}^{x}}=0
\end{aligned}
$$

with hybrid nuclear coordinate $(x)$ and external field $(n)$ perturbations. Here

$$
\begin{aligned}
\mathbf{F}^{n} & =\mathbf{M}_{n}+\mathscr{R}_{n} \mathscr{T}^{-1} \mathbf{V}+(\mathbf{I I}+\mathbf{\Omega}) \cdot \mathbf{P}^{n} \\
\mathbf{F}^{n x} & =\mathbf{M}_{n}^{[x]}+\mathscr{R}_{n} \mathscr{T}^{-1} \mathbf{V}^{[x]}+\left(\mathbf{I I}^{[x]}+\mathbf{\Omega}^{[x]}\right) \cdot \mathbf{P}^{n}+(\mathbf{I I}+\mathbf{\Omega}) \cdot \mathbf{P}^{n x}
\end{aligned}
$$

After moving $\mathbf{P}^{n x}$-dependent terms to one side of Eq. (B6) and keep only the vo block, we arrive at

$$
(\mathbf{A}+\mathbf{B}) \Theta_{\mathrm{vo}}^{[n x]}=\mathbf{L}_{\mathrm{vo}}^{[n x]}
$$

with

$$
\begin{aligned}
\mathbf{L}^{[n x]}= & -\left[\mathbf{F}, \mathbf{P}^{n}\right]_{\mathbf{S}^{x}}-\left[\mathbf{F}^{n}, \mathbf{P}\right]_{\mathbf{S}^{x}}-\left[\mathbf{F}^{[n x]}, \mathbf{P}\right]_{\mathbf{S}}-\left[\mathbf{F}, \mathbf{P}^{[n x]}\right]_{\mathbf{S}} \\
& -\left[\mathbf{F}^{n}, \mathbf{P}^{x}\right]_{\mathbf{S}}-\left[\mathbf{F}^{x}, \mathbf{P}^{n}\right]_{\mathbf{S}}
\end{aligned}
$$

Instead of explicitly solving the second-order response in Eq. B8, however, we can directly compute the desired product $\mathbf{P}_{\mathrm{vo}}^{x n} \cdot \tilde{\mathbf{M}}_{m, \text { vo }}$ in (28) as

$$
\Theta_{\mathrm{vo}}^{[n x]} \cdot\left(\tilde{\mathbf{M}}_{m}\right)_{\mathrm{vo}}=\left(\mathbf{L}_{\mathrm{vo}}^{[n x]} \cdot(\mathbf{A}+\mathbf{B})^{-1}\right) \cdot\left(\tilde{\mathbf{M}}_{m}\right)_{\mathrm{vo}}=\mathbf{L}_{\mathrm{vo}}^{[n x]} \cdot \Theta_{\mathrm{vo}}^{[m]}
$$

by interchanging the variables. It corresponds to the wellknown $2 n+1$ rule.

\footnotetext{
${ }^{1}$ S. Eustis and M. A. El-Sayed, "Why gold nanoparticles are more precious than pretty gold: Noble metal surface plasmon resonance and its enhancement of the radiative and nonradiative properties of nanocrystals of different shapes," Chem. Soc. Rev. 35, 209-217 (2006).

${ }^{2}$ S. A. Maier, Plasmonics: Fundamentals and Applications (Springer, New York, 2007).

${ }^{3}$ M. Pelton and G. W. Bryant, Introduction to Metal-Nanoparticle Plasmonics (Wiley: Science Wise Publishing, Hoboken, New Jersey, 2013).

${ }^{4}$ J. Langer, D. J. de Aberasturi, J. Aizpurua, R. A. Alvarez-Puebla, B. Auguié, J. J. Baumberg, G. C. Bazan, S. E. J. Bell, A. Boisen, A. G. Brolo, J. Choo, D. Cialla-May, V. Deckert, L. Fabris, K. Faulds, F. J. G. de Abajo, R. Goodacre, D. Graham, A. J. Haes, C. L. Haynes, C. Huck, T. Itoh, M. Käll, J. Kneipp, N. A. Kotov, H. Kuang, E. C. L. Ru, H. K. Lee, J.F. Li, X. Y. Ling, S. A. Maier, T. Mayerhöfer, M. Moskovits, K. Murakoshi, J.-M. Nam, S. Nie, Y. Ozaki, I. Pastoriza-Santos, J. Perez-Juste, J. Popp, A. Pucci, S. Reich, B. Ren, G. C. Schatz, T. Shegai, S. Schlücker, L.-L. Tay, K. G. Thomas, Z.-Q. Tian, R. P. V. Duyne, T. Vo-Dinh, Y. Wang, K. A. Willets, C. Xu, H. Xu, Y. Xu, Y. S. Yamamoto, B. Zhao, and L. M. LizMarzán, "Present and future of surface-enhanced Raman scattering," ACS Nano 14, 28-117 (2019).
}

${ }^{5}$ W. Hou, W. H. Hung, P. Pavaskar, A. Goeppert, M. Aykol, and S. B. Cronin, "Photocatalytic conversion of $\mathrm{CO}_{2}$ to hydrocarbon fuels via plasmonenhanced absorption and metallic interband transitions," ACS Catal. 1, 929-936 (2011).

${ }^{6}$ C. Novo, A. M. Funston, and P. Mulvaney, "Direct observation of chemical reactions on single gold nanocrystals using surface plasmon spectroscopy," Nat. Nanotechnol. 3, 598-602 (2008).

${ }^{7}$ R. F. Aroca, "Plasmon enhanced spectroscopy," Phys. Chem. Chem. Phys. 15, 5355 (2013).

${ }^{8}$ C. D. Geddes, "Metal-enhanced fluorescence," Phys. Chem. Chem. Phys. 15, 19537 (2013).

${ }^{9}$ J. Dong, Z. Zhang, H. Zheng, and M. Sun, "Recent progress on plasmonenhanced fluorescence," Nanophotonics 4, 472-490 (2015).

${ }^{10}$ J.-F. Li, C.-Y. Li, and R. F. Aroca, "Plasmon-enhanced fluorescence spectroscopy," Chem. Soc. Rev. 46, 3962-3979 (2017).

${ }^{11}$ M. J. Kale, T. Avanesian, H. Xin, J. Yan, and P. Christopher, "Controlling catalytic selectivity on metal nanoparticles by direct photoexcitation of adsorbate-metal bonds," Nano Lett. 14, 5405-5412 (2014).

${ }^{12}$ S. Linic, U. Aslam, C. Boerigter, and M. Morabito, "Photochemical transformations on plasmonic metal nanoparticles," Nat. Mater. 14, 567-576 (2015).

${ }^{13}$ U. Aslam, V. G. Rao, S. Chavez, and S. Linic, "Catalytic conversion of solar to chemical energy on plasmonic metal nanostructures," Nat. Catal. 1, 656-665 (2018).

${ }^{14}$ E. Kazuma and Y. Kim, "Mechanistic studies of plasmon chemistry on metal catalysts," Angew. Chem. Int. Ed. 58, 4800-4808 (2019).

${ }^{15}$ N. Valley, N. Greeneltch, R. P. V. Duyne, and G. C. Schatz, "A look at the origin and magnitude of the chemical contribution to the enhancement mechanism of surface-enhanced Raman spectroscopy (SERS): Theory and experiment," J. Phys. Chem. Lett. 4, 2599-2604 (2013).

${ }^{16}$ C. Boerigter, R. Campana, M. Morabito, and S. Linic, "Evidence and implications of direct charge excitation as the dominant mechanism in plasmonmediated photocatalysis," Nat. Commun. 7 (2016), 10.1038/ncomms 10545.

${ }^{17} \mathrm{Y}$. Yu, V. Sundaresan, and K. A. Willets, "Hot carriers versus thermal effects: Resolving the enhancement mechanisms for plasmon-mediated photoelectrochemical reactions," J. Phys. Chem. C 122, 5040-5048 (2018).

${ }^{18}$ V. Faessler, C. Hrelescu, A. Lutich, L. Osinkina, S. Mayilo, F. Jäckel, and J. Feldmann, "Accelerating fluorescence resonance energy transfer with plasmonic nanoresonators," Chem. Phys. Lett. 508, 67-70 (2011).

${ }^{19}$ P. Ghenuche, J. de Torres, S. B. Moparthi, V. Grigoriev, and J. Wenger, "Nanophotonic enhancement of the förster resonance energy-transfer rate with single nanoapertures," Nano Lett. 14, 4707-4714 (2014).

${ }^{20}$ L.-Y. Hsu, W. Ding, and G. C. Schatz, "Plasmon-coupled resonance energy transfer," J. Phys. Chem. Lett. 8, 2357-2367 (2017).

${ }^{21}$ W. Ding, L.-Y. Hsu, C. W. Heaps, and G. C. Schatz, "Plasmon-coupled resonance energy transfer II: Exploring the peaks and dips in the electromagnetic coupling factor," J. Phys. Chem. C 122, 22650-22659 (2018).

${ }^{22}$ J. Zhao, A. O. Pinchuk, J. M. McMahon, S. Li, L. K. Ausman, A. L. Atkinson, and G. C. Schatz, "Methods for describing the electromagnetic properties of silver and gold nanoparticles," Acc. Chem. Res. 41, 1710-1720 (2008).

${ }^{23}$ L. Jensen, C. M. Aikens, and G. C. Schatz, "Electronic structure methods for studying surface-enhanced Raman scattering," Chem. Soc. Rev. 37, 1061 (2008)

${ }^{24}$ S. M. Morton, D. W. Silverstein, and L. Jensen, "Theoretical studies of plasmonics using electronic structure methods," Chem. Rev. 111, 39623994 (2011).

${ }^{25}$ C. M. Aikens and G. C. Schatz, "TDDFT studies of absorption and SERS spectra of pyridine interacting with $\mathrm{Au}_{20}$," J. Phys. Chem. A 110, 13317 13324 (2006).

${ }^{26}$ J. C. Idrobo, W. Walkosz, S. F. Yip, S. Ögüt, J. Wang, and J. Jellinek, "Static polarizabilities and optical absorption spectra of gold clusters $\left(\mathrm{au}_{n}, n=2-14\right.$ and 20) from first principles," Phys. Rev. B 76 (2007), 10.1103/physrevb.76.205422.

${ }^{27}$ S. M. Morton and L. Jensen, "Understanding the molecule-surface chemical coupling in SERS," J. Am. Chem. Soc. 131, 4090-4098 (2009).

${ }^{28}$ L. Liu, D. Chen, H. Ma, and W. Liang, "Spectral characteristics of chemical enhancement on SERS of benzene-like derivatives: Franck-condon and herzberg-teller contributions," J. Phys. Chem. C 119, 27609-27619 (2015).

${ }^{29}$ M. J. Trujillo, S. L. Strausser, J. C. Becca, J. F. DeJesus, L. Jensen, D. M. 
Jenkins, and J. P. Camden, "Using SERS to understand the binding of nheterocyclic carbenes to gold surfaces," J. Phys. Chem. Lett. 9, 6779-6785 (2018).

${ }^{30} \mathrm{R}$. Chen and L. Jensen, "Interpreting the chemical mechanism in SERS using a Raman bond model," J. Chem. Phys. 152, 024126 (2020).

${ }^{31}$ R. Chen and L. Jensen, "Quantifying the enhancement mechanisms of surface-enhanced Raman scattering using a Raman bond model," J. Chem. Phys. 153, 224704 (2020).

${ }^{32}$ X.-G. Zhang, L. Zhang, S. Feng, H. Qin, D.-Y. Wu, and Y. Zhao, "Light driven mechanism of carbon dioxide reduction reaction to carbon monoxide on gold nanoparticles: A theoretical prediction," J. Phys. Chem. Lett. 12, $1125-1130$ (2021).

${ }^{33}$ J. Mullin, N. Valley, M. G. Blaber, and G. C. Schatz, "Combined quantum mechanics (TDDFT) and classical electrodynamics (Mie theory) methods for calculating surface enhanced Raman and hyper-Raman spectra," J. Phys. Chem. A 116, 9574-9581 (2012).

${ }^{34}$ W.-H. Yang, G. C. Schatz, and R. P. V. Duyne, "Discrete dipole approximation for calculating extinction and Raman intensities for small particles with arbitrary shapes," J. Chem. Phys. 103, 869-875 (1995).

${ }^{35}$ S. Vukovic, S. Corni, and B. Mennucci, "Fluorescence enhancement of chromophores close to metal nanoparticles. Optimal setup revealed by the polarizable continuum model," J. Phys. Chem. C 113, 121-133 (2008).

${ }^{36}$ Á. Sánchez-González, S. Corni, and B. Mennucci, "Surface-enhanced fluorescence within a metal nanoparticle array: The role of solvent and plasmon couplings," J. Phys. Chem. C 115, 5450-5460 (2011).

${ }^{37}$ O. Andreussi, S. Caprasecca, L. Cupellini, I. Guarnetti-Prandi, C. A. Guido, S. Jurinovich, L. Viani, and B. Mennucci, "Plasmon enhanced light harvesting: Multiscale modeling of the FMO protein coupled with gold nanoparticles," J. Phys. Chem. A 119, 5197-5206 (2014).

${ }^{38} \mathrm{~J}$. Applequist, "An atom dipole interaction model for molecular optical properties," Acc. Chem. Res. 10, 79-85 (1977).

${ }^{39}$ L. Jensen, P.-O. Åstrand, A. Osted, J. Kongsted, and K. V. Mikkelsen, "Polarizability of Molecular Clusters as Calculated by a Dipole Interaction Model," J. Chem. Phys. 116, 4001-4010 (2002).

${ }^{40}$ H. S. Smalø, P.-O. Åstrand, and A. Mayer, "Combined nonmetallic electronegativity equalisation and point-dipole interaction model for the frequency-dependent polarisability," Mol. Phys. 111, 1470-1481 (2013).

${ }^{41}$ N. Davari, S. Haghdani, P.-O. Åstrand, and G. C. Schatz, "Local electric field factors by a combined charge-transfer and point-dipole interaction model," RSC Adv. 5, 31594-31605 (2015).

${ }^{42} \mathrm{~A}$. Mayer, "Formulation in terms of normalized propagators of a chargedipole model enabling the calculation of the polarization properties of fullerenes and carbon nanotubes," Phys. Rev. B 75 (2007), 10.1103/PhysRevB.75.045407.

${ }^{43}$ A. Mayer, A. L. González, C. M. Aikens, and G. C. Schatz, "A chargedipole interaction model for the frequency-dependent polarizability of silver clusters," Nanotechnology 20, 195204 (2009).

${ }^{44}$ L. L. Jensen and L. Jensen, "Electrostatic interaction model for the calculation of the polarizability of large noble metal nanoclusters," J. Phys. Chem. C 112, 15697-15703 (2008).

${ }^{45}$ L. L. Jensen and L. Jensen, "Atomistic electrodynamics model for optical properties of silver nanoclusters," J. Phys. Chem. C 113, 15182-15190 (2009).

${ }^{46}$ X. Chen, J. E. Moore, M. Zekarias, and L. Jensen, "Atomistic electrodynamics simulations of bare and ligand-coated nanoparticles in the quantum size regime," Nat. Commun. 6 (2015), 10.1038/ncomms9921.

${ }^{47}$ J. Lim, S. Kang, J. Kim, W. Y. Kim, and S. Ryu, "Non-empirical atomistic dipole-interaction-model for quantum plasmon simulation of nanoparticles," Sci. Rep. 7 (2017), 10.1038/s41598-017-16053-6.

${ }^{48} \mathrm{~S}$. T. Olsen and K. V. Mikkelsen, "First hyperpolarizability of paraaminoaniline induced by a variety of gold nano particles," Phys. Chem. Chem. Phys. 18, 24343-24349 (2016).

${ }^{49}$ I. M. I. Boye, M. H. Hansen, and K. V. Mikkelsen, "The influence of nanoparticles on the polarizabilities and hyperpolarizabilities of photochromic molecules," Phys. Chem. Chem. Phys. 20, 23320-23327 (2018).

${ }^{50} \mathrm{~S}$. M. Morton and L. Jensen, "A discrete interaction model/quantum mechanical method for describing response properties of molecules adsorbed on metal nanoparticles," J. Chem. Phys. 133, 074103 (2010).

${ }^{51}$ S. M. Morton and L. Jensen, "A discrete interaction model/quantum mechanical method to describe the interaction of metal nanoparticles and molecular absorption," J. Chem. Phys. 135, 134103 (2011).

${ }^{52}$ J. L. Payton, S. M. Morton, J. E. Moore, and L. Jensen, “A discrete interaction model/quantum mechanical method for simulating surface-enhanced Raman spectroscopy," J. Chem. Phys. 136, 214103 (2012).

${ }^{53}$ J. M. Rinaldi, S. M. Morton, and L. Jensen, "A discrete interaction model/quantum mechanical method for simulating nonlinear optical properties of molecules near metal surfaces," Mol. Phys. 111, 1322-1331 (2013).

${ }^{54}$ Z. Rinkevicius, X. Li, J. A. R. Sandberg, K. V. Mikkelsen, and H. Ågren, "A hybrid density functional theory/molecular mechanics approach for linear response properties in heterogeneous environments," J. Chem. Theory Comput. 10, 989-1003 (2014).

${ }^{55}$ A. Fihey, F. Maurel, and A. Perrier, "Plasmon-excitation coupling for dithienylethene/gold nanoparticle hybrid systems: A theoretical study," J. Phys. Chem. C 119, 9995-10006 (2015).

${ }^{56} \mathrm{Z}$. Hu and L. Jensen, "A discrete interaction model/quantum mechanical method for simulating plasmon-enhanced two-photon absorption," J. Chem. Theory Comput. 14, 5896-5903 (2018).

${ }^{57}$ J. L. Payton, S. M. Morton, J. E. Moore, and L. Jensen, "A hybrid atomistic electrodynamics-quantum mechanical approach for simulating surfaceenhanced Raman scattering," Acc. Chem. Res. 47, 88-99 (2014).

${ }^{58} \mathrm{Z}$. Hu, D. V. Chulhai, and L. Jensen, "Simulating surface-enhanced hyperRaman scattering using atomistic electrodynamics-quantum mechanical models," J. Chem. Theory Comput. 12, 5968-5978 (2016).

${ }^{59}$ J. C. Becca, X. Chen, and L. Jensen, "A discrete interaction model/quantum mechanical method for simulating surface-enhanced Raman spectroscopy in solution," J. Chem. Phys. 154, 224705 (2021).

${ }^{60}$ N. Chiang, N. Jiang, D. V. Chulhai, E. A. Pozzi, M. C. Hersam, L. Jensen, T. Seideman, and R. P. V. Duyne, "Molecular-resolution interrogation of a porphyrin monolayer by ultrahigh vacuum tip-enhanced Raman and fluorescence spectroscopy," Nano Lett. 15, 4114-4120 (2015).

${ }^{61}$ T. Giovannini, A. Puglisi, M. Ambrosetti, and C. Cappelli, "Polarizable QM/MM approach with fluctuating charges and fluctuating dipoles: The QM/FQF $\mu$ model," J. Chem. Theory Comput. 15, 2233-2245 (2019).

${ }^{62}$ T. Giovannini, R. R. Riso, M. Ambrosetti, A. Puglisi, and C. Cappelli, "Electronic transitions for a fully polarizable QM/MM approach based on fluctuating charges and fluctuating dipoles: Linear and corrected linear response regimes," J. Chem. Phys. 151, 174104 (2019).

${ }^{63}$ M. J. Frisch, Y. Yamaguchi, J. F. Gaw, H. F. Schaefer, and J. S. Binkley, "Analytic Raman intensities from molecular electronic wave functions," J. Chem. Phys. 84, 531-532 (1986).

${ }^{64}$ M. Frisch, M. Head-Gordon, and J. Pople, "Direct analytic SCF second derivatives and electric field properties," Chem. Phys. 141, 189-196 (1990).

${ }^{65} \mathrm{O}$. Quinet and B. Champagne, "Time-dependent hartree-fock schemes for analytical evaluation of the Raman intensities," J. Chem. Phys. 115, $6293-$ 6299 (2001).

${ }^{66}$ T. Giovannini, L. Grazioli, M. Ambrosetti, and C. Cappelli, "Calculation of IR spectra with a fully polarizable QM/MM approach based on fluctuating charges and fluctuating dipoles," J. Chem. Theory Comput. 15, 5495-5507 (2019).

${ }^{67}$ T. Giovannini, M. Olszówka, F. Egidi, J. R. Cheeseman, G. Scalmani, and C. Cappelli, "Polarizable embedding approach for the analytical calculation of Raman and Raman optical activity spectra of solvated systems," J. Chem. Theory Comput. 13, 4421-4435 (2017).

${ }^{68}$ K. O. H. M. Dundas, M. T. P. Beerepoot, M. Ringholm, S. Reine, R. Bast, N. H. List, J. Kongsted, K. Ruud, and J. M. H. Olsen, "Harmonic infrared and Raman spectra in molecular environments using the polarizable embedding model," J. Chem. Theory Comput. 17, 3599-3617 (2021).

${ }^{69}$ F. Liu, Z. Gan, Y. Shao, C.-P. Hsu, A. Dreuw, M. Head-Gordon, B. T. Miller, B. R. Brooks, J.-G. Yu, T. R. Furlani, and J. Kong, "A parallel implementation of the analytic nuclear gradient for time-dependent density functional theory within the tamm-dancoff approximation," Mol. Phys. 108, 2791-2800 (2010).

${ }^{70}$ E. B. Wilson, J. C. Decius, and P. C. Cross, Molecular vibrations: The theory of infrared and Raman vibrational spectra (Courier Corporation, 1980).

${ }^{71}$ D. Porezag and M. R. Pederson, "Infrared intensities and Raman-scattering activities within density-functional theory," Phys. Rev. B 54, 7830-7836 (1996).

${ }^{72} \mathrm{~F}$. Furche, "On the density matrix based approach to time-dependent density functional response theory," J. Chem. Phys. 114, 5982-5992 (2001). 
${ }^{73}$ S. M. Parker, D. Rappoport, and F. Furche, "Quadratic response properties from TDDFT: Trials and tribulations," J. Chem. Theory Comput. 14, 807819 (2017).

${ }^{74}$ E. Epifanovsky, A. T. B. Gilbert, X. Feng, J. Lee, Y. Mao, N. Mardirossian, P. Pokhilko, A. F. White, M. P. Coons, A. L. Dempwolff, Z. Gan, D. Hait, P. R. Horn, L. D. Jacobson, I. Kaliman, J. Kussmann, A. W. Lange, K. U. Lao, D. S. Levine, J. Liu, S. C. McKenzie, A. F. Morrison, K. D. Nanda, F. Plasser, D. R. Rehn, M. L. Vidal, Z.-Q. You, Y. Zhu, B. Alam, B. J. Albrecht, A. Aldossary, E. Alguire, J. H. Andersen, V. Athavale, D. Barton, K. Begam, A. Behn, N. Bellonzi, Y. A. Bernard, E. J. Berquist, H. G. A. Burton, A. Carreras, K. Carter-Fenk, R. Chakraborty, A. D. Chien, K. D. Closser, V. Cofer-Shabica, S. Dasgupta, M. de Wergifosse, J. Deng, M. Diedenhofen, H. Do, S. Ehlert, P.-T. Fang, S. Fatehi, Q. Feng, T. Friedhoff, J. Gayvert, Q. Ge, G. Gidofalvi, M. Goldey, J. Gomes, C. E. GonzálezEspinoza, S. Gulania, A. O. Gunina, M. W. D. Hanson-Heine, P. H. P. Harbach, A. Hauser, M. F. Herbst, M. Hernández Vera, M. Hodecker, Z. C. Holden, S. Houck, X. Huang, K. Hui, B. C. Huynh, M. Ivanov, Á. Jász, H. Ji, H. Jiang, B. Kaduk, S. Köhler, K. Khistyaev, J. Kim, G. Kis, P. Klunzinger, Z. Koczor-Benda, J. H. Koh, D. Kosenkov, L. Koulias, T. Kowalczyk, C. M. Krauter, K. Kue, A. Kunitsa, T. Kus, I. Ladjánszki, A. Landau, K. V. Lawler, D. Lefrancois, S. Lehtola, R. R. Li, Y.-P. Li, J. Liang, M. Liebenthal, H.-H. Lin, Y.-S. Lin, F. Liu, K.-Y. Liu, M. Loipersberger, A. Luenser, A. Manjanath, P. Manohar, E. Mansoor, S. F. Manzer, S.P. Mao, A. V. Marenich, T. Markovich, S. Mason, S. A. Maurer, P. F. McLaughlin, M. F. S. J. Menger, J.-M. Mewes, S. A. Mewes, P. Morgante, J. W. Mullinax, K. J. Oosterbaan, G. Paran, A. C. Paul, S. K. Paul, F. Pavošević, Z. Pei, S. Prager, E. I. Proynov, Á. Rák, E. Ramos-Cordoba, B. Rana, A. E. Rask, A. Rettig, R. M. Richard, F. Rob, E. Rossomme, T. Scheele, M. Scheurer, M. Schneider, N. Sergueev, S. M. Sharada, W. Skomorowski, D. W. Small, C. J. Stein, Y.-C. Su, E. J. Sundstrom, Z. Tao, J. Thirman, G. J. Tornai, T. Tsuchimochi, N. M. Tubman, S. P. Veccham, O. Vydrov, J. Wenzel, J. Witte, A. Yamada, K. Yao, S. Yeganeh, S. R. Yost, A. Zech, I. Y. Zhang, X. Zhang, Y. Zhang, D. Zuev, A. AspuruGuzik, A. T. Bell, N. A. Besley, K. B. Bravaya, B. R. Brooks, D. Casanova, J.-D. Chai, S. Coriani, C. J. Cramer, G. Cserey, A. E. DePrince, R. A. DiStasio, A. Dreuw, B. D. Dunietz, T. R. Furlani, W. A. Goddard, S. HammesSchiffer, T. Head-Gordon, W. J. Hehre, C.-P. Hsu, T.-C. Jagau, Y. Jung, A. Klamt, J. Kong, D. S. Lambrecht, W. Liang, N. J. Mayhall, C. W. McCurdy, J. B. Neaton, C. Ochsenfeld, J. A. Parkhill, R. Peverati, V. A. Rassolov, Y. Shao, L. V. Slipchenko, T. Stauch, R. P. Steele, J. E. Subotnik, A. J. W. Thom, A. Tkatchenko, D. G. Truhlar, T. Van Voorhis, T. A. Wesolowski, K. B. Whaley, H. L. Woodcock, P. M. Zimmerman, S. Faraji, P. M. W. Gill, M. Head-Gordon, J. M. Herbert, and A. I. Krylov, "Software for the frontiers of quantum chemistry: An overview of developments in the Q-Chem 5 package," J. Chem. Phys. 155, 084801 (2021).

${ }^{75}$ S.-W. Joo, "Surface-enhanced raman scattering of 4,4/-bipyridine on gold nanoparticle surfaces," Vib. Spectrosc. 34, 269-272 (2004).

${ }^{76}$ S.-W. Joo, "Adsorption of bipyridine compounds on gold nanoparticle surfaces investigated by UV-vis absorbance spectroscopy and surface enhanced raman scattering," Spectrosc. Lett. 39, 85-96 (2006).
${ }^{77}$ Z. Zhuang, J. Cheng, X. Wang, B. Zhao, X. Han, and Y. Luo, "Surfaceenhanced raman spectroscopy and density functional theory study on 4,4/bipyridine molecule," Spectrochim. Acta A Mol. Biomol. Spectrosc. 67, 509-516 (2007).

${ }^{78}$ Z. Zhuang, W. Ruan, N. Ji, X. Shang, X. Wang, and B. Zhao, "Surfaceenhanced raman scattering of 4,4/-bipyridine on silver by density functional theory calculations," Vib. Spectrosc. 49, 118-123 (2009).

${ }^{79}$ P. M. Gill, B. G. Johnson, and J. A. Pople, "A standard grid for density functional calculations," Chem. Phys. Lett. 209, 506-512 (1993).

${ }^{80}$ J. Neugebauer, M. Reiher, C. Kind, and B. A. Hess, "Quantum chemical calculation of vibrational spectra of large molecules?raman and IR spectra for buckminsterfullerene," J. Comput. Chem. 23, 895-910 (2002).

${ }^{81}$ M. D. Williams, D. S. Bradshaw, and D. L. Andrews, "Raman scattering mediated by neighboring molecules," J. Chem. Phys. 144, 174304 (2016).

${ }^{82}$ M. D. Williams, D. S. Bradshaw, and D. L. Andrews, "Symmetry analysis of Raman scattering mediated by neighboring molecules," J. Chem. Phys. 145, 184301 (2016)

${ }^{83}$ I. Russier-Antoine, F. Bertorelle, M. Vojkovic, D. Rayane, E. Salmon, C. Jonin, P. Dugourd, R. Antoine, and P.-F. Brevet, "Non-linear optical properties of gold quantum clusters. The smaller the better," Nanoscale 6 , 13572-13578 (2014).

${ }^{84} \mathrm{~K}$. Wu, J. Li, and C. Lin, "Remarkable second-order optical nonlinearity of nano-sized au20 cluster: a TDDFT study," Chem. Phys. Lett. 388, 353-357 (2004).

${ }^{85}$ R. S. Mulliken, "Electronic population analysis on LCAO-MO molecular wave functions. i," J. Chem. Phys. 23, 1833-1840 (1955).

${ }^{86}$ C. M. Breneman and K. B. Wiberg, "Determining atom-centered monopoles from molecular electrostatic potentials. The need for high sampling density in formamide conformational analysis," J. Comput. Chem. 11, 361-373 (1990)

${ }^{87}$ F. A. Momany, "Determination of partial atomic charges from ab initio molecular electrostatic potentials. Application to formamide, methanol, and formic acid," J. Phys. Chem. 82, 592-601 (1978).

${ }^{88}$ J. Řezáč and A. de la Lande, "Robust, basis-set independent method for the evaluation of charge-transfer energy in noncovalent complexes," J. Chem. Theory Comput. 11, 528-537 (2015).

${ }^{89}$ G. S. Kedziora and G. C. Schatz, "Calculating dipole and quadrupole polarizabilities relevant to surface enhanced raman spectroscopy," Spectrochim. Acta A Mol. Biomol. Spectrosc. 55, 625-638 (1999).

${ }^{90}$ J. Yang, Z. Pei, J. Deng, Y. Mao, Q. Wu, Z. Yang, B. Wang, C. M. Aikens, W. Liang, and Y. Shao, "Analysis and visualization of energy densities. I. Insights from real-time time-dependent density functional theory simulations," Phys. Chem. Chem. Phys. 22, 26838-26851 (2020).

${ }^{91}$ F. Liu, Z. Gan, Y. Shao, C.-P. Hsu, A. Dreuw, M. Head-Gordon, B. T. Miller, B. R. Brooks, J.-G. Yu, T. R. Furlani, and J. Kong, "A parallel implementation of the analytic nuclear gradient for time-dependent density functional theory within the Tamm-Dancoff approximation," Mol. Phys. 108, 2791-2800 (2010). 